\title{
ManlyMix: An R Package for Manly Mixture Modeling
}

by Xuwen Zhu, Volodymyr Melnykov

\begin{abstract}
Model-based clustering is a popular technique for grouping objects based on a finite mixture model. It has countless applications in different fields of study. The R package ManlyMix implements the Manly mixture model that allows modeling skewness within data groups and performs cluster analysis. ManlyMix is a powerful diagnostics tool that is capable of conducting investigation concerning the normality of variables upon fitting of a Manly forward or backward model. Theoretical foundations as well as description of functions are provided. All features of the package are illustrated with examples in great detail. The analysis of real-life datasets demonstrates the flexibility and usefulness of the package.
\end{abstract}

\section{Introduction}

Finite mixture models provide a powerful tool to model heterogeneous data. Their flexibility, close connection to cluster analysis, and interpretability make them increasingly appealing to researchers and practitioners these days. The applications of finite mixture modeling can be found in all fields, including medicine (Schlattmann, 2009), transportation (Park and Lord, 2009), dendrochronology (Michael and Melnykov, 2016), and environment science (Gillespie and Neale, 2006), just to name a few.

The Bayes decision rule, applied to posterior probabilities obtained in the course of fitting a mixture model, yields a clustering result. Such a procedure is called model-based clustering. It assumes the existence of a one-to-one correspondence between each distribution in the mixture model and underlying data group.

If all components in the model are Gaussian distributions, the mixture is called a Gaussian mixture model. Gaussian mixtures are very popular among practitioners due to their interpretability and simplicity. However, when there is severe skewness in data, Gaussian mixtures models do not provide a good fit to the data. As a result, model-based clustering might produce unsatisfactory results. In such cases, more flexible mixtures should be adopted. Some existing software packages that provide such functionality are listed in Table 1. Here, mixsmsn, EMMIXskew, and EMMIXuskew packages are based on skew-normal and skew- $t$ distributions, which are popular choices for modeling skewed data. On the other hand, flowClust is the only package that implements a transformation-based mixture model. It relies on the celebrated Box-Cox transformation to near-normality applied to all dimensions within the same mixture component. This leads to extra $K$ parameters $\lambda_{k}$ in the resulting mixture. The package is shown to model flow cytometry data effectively. In many applications, however, it is reasonable to assume that transformation parameters can vary not only from component to component but also from variable to variable. In this paper, we introduce the R package ManlyMix (Zhu and Melnykov, 2016b), which provides readers with an alternative approach to modeling and clustering skewed data. Manly mixture models (Zhu and Melnykov, 2016a) are constructed based on the Manly back-transformation applied to each variable in multivariate Gaussian components.

The ManlyMix package implements several functions associated with Manly mixture models including the core function for running the EM algorithm, the forward and backward model selection procedure for eliminating unnecessary transformation parameters, and the Manly K-means algorithm, which serves as an extension of the traditional $K$-means. Other capabilities of the package include computing a Manly mixture overlap, simulating datasets from a Manly mixture, constructing density or contour plots for a fitted model, and assessing the variability of estimated parameters. The highlights of ManlyMix include:

\begin{tabular}{ll}
\hline Package & Mixture components \\
\hline flowClust (Lo et al., 2009) & $t$ mixture with Box-Cox transformation \\
mixsmsn (Prates et al., 2013) & scale skew-normal and skew- $t$ \\
EMMIXskew (Wang et al., 2013) & restricted skew-normal and skew- $t$ \\
EMMIXuskew (Lee and McLachlan, 2014) & unrestricted skew- $t$ \\
\hline
\end{tabular}

Table 1: Existing R packages for mixture modeling of skewed data. 
- providing an alternative approach to modeling heterogeneous skewed data;

- calling core functions from $\mathrm{C}$ for speed;

- providing excellent model interpretability through output of skewness parameters;

- preventing overfitting of the data by implementing model selection algorithms;

- offering effective assessment of mixture characteristics through the overlap calculation and variability assessment.

This paper is organized in the following way. A brief introduction to the Expectation-Maximization (EM) algorithm for Manly mixture models as well as the classification Expectation-Maximization (CEM) algorithm for Manly K-means is provided in the second section. In section "Package functionality and illustrative examples", a comprehensive description of all functions in ManlyMix is given along with the analysis of two real-life datasets. All features of the package are illustrated in great detail. Demo examples are constructed in section four for users to conduct further investigation of ManlyMix. In the last section, we provide a brief summary for the paper.

\section{Methodological and algorithmic details}

\section{Manly mixture model}

Consider a dataset $\boldsymbol{X}_{1}, \ldots, \boldsymbol{X}_{n}$ of size $n$, where $\boldsymbol{X}_{i}$ 's are $p$-variate independent observations that are identically distributed. The exponential (Manly) transformation to near normality is defined by

$$
\mathcal{M}(\boldsymbol{X} ; \boldsymbol{\lambda})=\left(\frac{e^{\lambda_{1} X_{1}}-1}{\lambda_{1}}, \ldots, \frac{e^{\lambda_{p} X_{p}}-1}{\lambda_{p}}\right)^{T},
$$

where the distribution of $\mathcal{M}\left(X ; \lambda_{k}\right)$ can be effectively approximated by multivariate normal distribution for an appropriate choice of $\lambda$ (Manly, 1976). $\mathcal{M}^{-1}$ represents the Manly back-transformation. This leads to a so-called Manly mixture model given by

$$
g(x ; \Psi)=\sum_{k=1}^{K} \tau_{k} \phi\left(\mathcal{M}\left(x ; \lambda_{k}\right) ; \boldsymbol{\mu}_{k}, \Sigma_{k}\right) \exp \left\{\lambda_{k}^{T} \boldsymbol{x}\right\},
$$

where $K$ is the number of components in the model, $\tau_{k}$ 's are mixing proportions such that $\sum_{k=1}^{K} \tau_{k}=1$, and $\lambda_{k}=\left(\lambda_{k 1}, \ldots, \lambda_{k p}\right)^{T}$ is a $p$-dimensional skewness vector which controls the transformation of the $k$ th component. $\phi\left(\cdot ; \mu_{k}, \Sigma_{k}\right)$ is the p-variate normal probability density function. $\boldsymbol{\mu}_{k}$ and $\boldsymbol{\Sigma}_{k}$ are the mean vector and variance-covariance matrix of the $k$ th component after transformation. $\Psi$, as the entire parameter vector, includes $\tau_{k}{ }^{\prime} \mathrm{s}, \boldsymbol{\mu}_{k}{ }^{\prime} \mathrm{s}$ and $\boldsymbol{\Sigma}_{k}{ }^{\prime} \mathrm{s}$.

To find the MLE of the parameter vector $\Psi$, the Expectation-Maximization (EM) algorithm (Dempster et al., 1977; McLachlan and Krishnan, 2008) needs to be employed. Each iteration of the EM algorithm consists of two steps, the E-step and M-step. Let $s$ denote the iteration number. The E-step computes the posterior probabilities

$$
\pi_{i k}^{(s)}=\frac{\tau_{k}^{(s-1)} \phi\left(\mathcal{M}\left(\boldsymbol{x}_{i} ; \boldsymbol{\lambda}_{k}^{(s-1)}\right) ; \boldsymbol{\mu}_{k}^{(s-1)}, \Sigma_{k}^{(s-1)}\right) \exp \left\{\left(\boldsymbol{\lambda}_{k}^{(s-1)}\right)^{T} \boldsymbol{x}_{i}\right\}}{\sum_{k^{\prime}=1}^{K} \tau_{k^{\prime}}^{(s-1)} \phi\left(\mathcal{M}\left(\boldsymbol{x}_{i} ; \boldsymbol{\lambda}_{k^{\prime}}^{(s-1)}\right) ; \boldsymbol{\mu}_{k^{\prime}}^{(s-1)}, \boldsymbol{\Sigma}_{k^{\prime}}^{(s-1)}\right) \exp \left\{\left(\boldsymbol{\lambda}_{k^{\prime}}^{(s-1)}\right)^{T} \boldsymbol{x}_{i}\right\}}
$$

based on the the parameter vector from the previous step, $\mathbf{\Psi}^{(s-1)}$. The M-step updates the parameters in each iteration. The closed-form expressions are available for the parameters $\tau_{k}^{(s)}, \boldsymbol{\mu}_{k}^{(s)}, \boldsymbol{\Sigma}_{k}^{(s)}$ and are given by

$$
\begin{aligned}
\tau_{k}^{(s)} & =\frac{\sum_{i=1}^{n} \pi_{i k}^{(s)}}{n}, \quad \boldsymbol{\mu}_{k}^{(s)}=\frac{\sum_{i=1}^{n} \pi_{i k}^{(s)} \mathcal{M}\left(\boldsymbol{x}_{i} ; \lambda_{k}^{(s)}\right)}{\sum_{i=1}^{n} \pi_{i k}^{(s)}}, \quad \text { and } \\
\Sigma_{k}^{(s)} & =\frac{\sum_{i=1}^{n} \pi_{i k}^{(s)}\left(\mathcal{M}\left(\boldsymbol{x}_{i} ; \lambda_{k}^{(s)}\right)-\boldsymbol{\mu}_{k}^{(s)}\right)\left(\mathcal{M}\left(\boldsymbol{x}_{i} ; \lambda_{k}^{(s)}\right)-\boldsymbol{\mu}_{k}^{(s)}\right)^{T}}{\sum_{i=1}^{n} \pi_{i k}^{(s)}} .
\end{aligned}
$$


For $\lambda_{k}$, closed-form solution is not available and Nelder-Mead numerical optimization of the function

$$
\begin{aligned}
Q_{k}\left(\boldsymbol{\lambda}_{k} \mid \Psi^{(s)}\right)\left(\boldsymbol{\lambda}_{k}\right) & =\sum_{i=1}^{n} \pi_{i k}^{(s)}\left\{\operatorname { l o g } \phi \left(\mathcal{M}\left(\boldsymbol{x}_{i} ; \boldsymbol{\lambda}_{k}\right) ; \sum_{i=1}^{n} \pi_{i k}^{(s)} \mathcal{M}\left(\boldsymbol{x}_{i} ; \lambda_{k}\right) / \sum_{i=1}^{n} \pi_{i k}^{(s)}\right.\right. \\
& \left.\sum_{i=1}^{n} \pi_{i k}^{(s)}\left(\mathcal{M}\left(\boldsymbol{x}_{i} ; \lambda_{k}\right)-\frac{\sum_{i=1}^{n} \pi_{i k}^{(s)} \mathcal{M}\left(\boldsymbol{x}_{i} ; \boldsymbol{\lambda}_{k}\right)}{\sum_{i=1}^{n} \pi_{i k}^{(s)}}\right)\left(\mathcal{M}\left(\boldsymbol{x}_{i} ; \boldsymbol{\lambda}_{k}\right)-\frac{\sum_{i=1}^{n} \pi_{i k}^{(s)} \mathcal{M}\left(\boldsymbol{x}_{i} ; \boldsymbol{\lambda}_{k}\right)}{\sum_{i=1}^{n} \pi_{i k}^{(s)}}\right)^{T}\right) \\
& \left.+\lambda_{k}{ }^{T} \boldsymbol{x}_{i}\right\}+\mathrm{const}
\end{aligned}
$$

gives us the estimated parameter vector.

The EM algorithm could be started with an initial partition of the data passed into the M-step. Or the E-step is run first with initial parameters $\tau_{k}^{(0)}, \boldsymbol{\mu}_{k}^{(0)}, \Sigma_{k}^{(0)}, \lambda_{k}^{(0)}$. The algorithm stops when the convergence criterion is met. In the R package ManlyMix, we monitor the relative difference between Q-function values from two consecutive steps. If it is smaller than a user specified tolerance level, $1 e-5$ by default, the algorithm stops. This is a speedier choice due to the fact that Q-function values are immediately available after numeric optimization of Equation 4. Such criterion is similar to monitoring the relative difference between log-likelihood values. Upon convergence, the Bayes decision rule assigns each observation to its cluster according to the maximized posterior probabilities from the last E-step. The estimated label of the $i$ th observation is given by

$$
\hat{Z}_{i}=\operatorname{argmax}_{k} \hat{\pi}_{i k} .
$$

In ManlyMix, the function Manly. EM() runs the EM algorithm for a Manly mixture model and returns estimated model parameters, posterior probabilities, as well as a classification vector. This function is constructed in $\mathrm{C}$ for computational efficiency.

\section{Pairwise overlap}

Pairwise overlap, introduced by Maitra and Melnykov (2010), is a measure of the interaction between two mixture components. If we denote $\omega_{k_{1}, k_{2}}$ as the pairwise overlap of components $k_{1}$ and $k_{2}$, it is defined as the sum of two misclassification probabilities

$$
\omega_{k_{1}, k_{2}}=\omega_{k_{1} \mid k_{2}}+\omega_{k_{2} \mid k_{1}}
$$

where $\omega_{k_{1} \mid k_{2}}$ represents the probability that a random variable $X$ is mistakenly classified to group $k_{1}$ while it came from the component $k_{2}$. For a Manly mixture, $\omega_{k_{1} \mid k_{2}}$ can be written as

$$
\begin{aligned}
\omega_{k_{1} \mid k_{2}}=\operatorname{Pr}\left[\frac{\phi\left(\mathcal{M}\left(\boldsymbol{X} ; \boldsymbol{\lambda}_{k_{1}}\right) ; \boldsymbol{\mu}_{k_{1}}, \boldsymbol{\Sigma}_{k_{1}}\right) \exp \left\{\boldsymbol{\lambda}_{k_{1}}^{T} \boldsymbol{X}\right\}}{\phi\left(\mathcal{M}\left(\boldsymbol{X} ; \boldsymbol{\lambda}_{k_{2}}\right) ; \boldsymbol{\mu}_{k_{2}}, \boldsymbol{\Sigma}_{k_{2}}\right) \exp \left\{\boldsymbol{\lambda}_{k_{2}}^{T} \boldsymbol{X}\right\}}>\frac{\tau_{k_{2}}}{\tau_{k_{1}}} \mid \boldsymbol{X} \sim \phi\left(\mathcal{M}\left(\boldsymbol{x} ; \boldsymbol{\lambda}_{k_{2}}\right) ; \boldsymbol{\mu}_{k_{2}}, \boldsymbol{\Sigma}_{k_{2}}\right) \exp \left\{\boldsymbol{\lambda}_{k_{2}}^{T} \boldsymbol{x}\right\}\right] \\
=\operatorname{Pr}\left[-\frac{1}{2}\left(\mathcal{M}\left(\mathcal{M}^{-1}\left(\boldsymbol{Y} ; \boldsymbol{\lambda}_{k_{2}}\right) ; \boldsymbol{\lambda}_{k_{1}}\right)-\boldsymbol{\mu}_{k_{1}}\right)^{T} \boldsymbol{\Sigma}_{k_{1}}^{-1}\left(\mathcal{M}\left(\mathcal{M}^{-1}\left(\boldsymbol{Y} ; \boldsymbol{\lambda}_{k_{2}}\right) ; \boldsymbol{\lambda}_{k_{1}}\right)-\boldsymbol{\mu}_{k_{1}}\right)+\boldsymbol{\lambda}_{k_{1}}^{T} \mathcal{M}^{-1}\left(\boldsymbol{Y} ; \boldsymbol{\lambda}_{k_{2}}\right)\right. \\
\left.+\frac{1}{2}\left(\boldsymbol{Y}-\boldsymbol{\mu}_{k_{2}}\right)^{T} \boldsymbol{\Sigma}_{k_{2}}^{-1}\left(\boldsymbol{Y}-\boldsymbol{\mu}_{k_{2}}\right)-\boldsymbol{\lambda}_{k_{2}}^{T} \mathcal{M}^{-1}\left(\boldsymbol{Y} ; \boldsymbol{\lambda}_{k_{2}}\right)>\log \left(\frac{\tau_{k_{2}}\left|\boldsymbol{\Sigma}_{k_{1}}\right|^{1 / 2}}{\tau_{k_{1}}\left|\boldsymbol{\Sigma}_{k_{2}}\right|^{1 / 2}}\right) \mid \boldsymbol{Y} \sim M V N\left(\boldsymbol{\mu}_{k_{2}}, \boldsymbol{\Sigma}_{k_{2}}\right)\right] .
\end{aligned}
$$

In ManlyMix, function Manly.overlap() estimates $\omega_{k_{1} \mid k_{2}}$ by sampling from corresponding distributions.

\section{Variability assessment}

The variability assessment of parameter estimates from Manly mixture model can be made by taking the inverse of the empirical observed information matrix $\boldsymbol{I}_{e}(\hat{\mathbf{\Psi}})$ (McLachlan and Basford, 1988) given by

$$
\boldsymbol{I}_{e}(\hat{\mathbf{\Psi}})=\sum_{i=1}^{n} \nabla q_{i}(\hat{\mathbf{\Psi}}) \nabla q_{i}^{T}(\hat{\mathbf{\Psi}}),
$$


where $q_{i}(\Psi)=\sum_{k=1}^{K} \pi_{i k}\left[\log \tau_{k}+\log \phi\left(\mathcal{M}\left(\boldsymbol{x}_{i} ; \boldsymbol{\lambda}_{k}\right) ; \boldsymbol{\mu}_{k}, \boldsymbol{\Sigma}_{k}\right)+\boldsymbol{\lambda}_{k} \boldsymbol{x}_{i}\right]$ and $\nabla$ stands for the gradient operator. We take partial derivatives in the gradient vector $\nabla q_{i}(\Psi)$ and obtain

$$
\begin{gathered}
\frac{\partial q_{i}(\boldsymbol{\Psi})}{\partial \tau_{k}}=\frac{\pi_{i k}}{\tau_{k}}-\frac{\pi_{i K}}{\tau_{K}}, \quad \frac{\partial q_{i}(\mathbf{\Psi})}{\partial \boldsymbol{\mu}_{k}}=\pi_{i k} \boldsymbol{\Sigma}_{k}^{-1}\left(\mathcal{M}\left(\boldsymbol{x}_{i} ; \boldsymbol{\lambda}_{k}\right)-\boldsymbol{\mu}_{k}\right), \\
\frac{\partial q_{i}(\boldsymbol{\Psi})}{\partial \operatorname{vech}\left\{\boldsymbol{\Sigma}_{k}\right\}}=\boldsymbol{G}^{T} \operatorname{vec}\left\{\frac{\pi_{i k}}{2} \boldsymbol{\Sigma}_{k}^{-1}\left(\left(\mathcal{M}\left(\boldsymbol{x}_{i} ; \boldsymbol{\lambda}_{k}\right)-\boldsymbol{\mu}_{k}\right)\left(\mathcal{M}\left(\boldsymbol{x}_{i} ; \boldsymbol{\lambda}_{k}\right)-\boldsymbol{\mu}_{k}\right)^{T} \boldsymbol{\Sigma}_{k}^{-1}-\boldsymbol{I}_{p}\right)\right\}, \\
\frac{\partial q_{i}(\boldsymbol{\Psi})}{\partial \boldsymbol{\lambda}_{k}}=-\pi_{i k} \boldsymbol{D}_{k} \boldsymbol{\Sigma}_{k}^{-1}\left(\mathcal{M}\left(\boldsymbol{x}_{i} ; \boldsymbol{\lambda}_{k}\right)-\boldsymbol{\mu}_{k}\right)+\pi_{i k} \boldsymbol{x}_{i},
\end{gathered}
$$

where $I_{p}$ is the identity matrix of size $p, \operatorname{vech}\{\cdot\}$ operator extracts the unique elements out of a symmetric $p \times p$ matrix and constructs a vector of length $p(p+1) / 2$. $G$ is a matrix with zero's and one's that enables the adoption of unique elements in a symmetric matrix (Melnykov, 2013). vec $\{\cdot\}$ is an operator which lines up all columns of a matrix one by one to form a vector. Finally,

$$
\boldsymbol{D}_{k}=\operatorname{diag}\left\{\left(1+\left(x_{i 1} \lambda_{k 1}-1\right) e^{\lambda_{k 1} x_{i 1}}\right) / \lambda_{k 1}^{2}, \ldots,\left(1+\left(x_{i p} \lambda_{k p}-1\right) e^{\lambda_{k p} x_{i p}}\right) / \lambda_{k p}^{2}\right\}
$$

The estimated covariance matrix can be found as $I_{e}^{-1}(\hat{\mathbf{Y}})$, i.e., by inverting the information matrix. Function Manly. $\operatorname{var}()$ in the package calculates the covariance matrix based on an estimated model provided by function Manly. EM().

\section{Forward and backward selection}

In the Manly mixture model, there are $K \times p$ skewness parameters $\lambda_{k j}$ corresponding to $K$ components and $p$ variables. Such a mixture is called a full Manly mixture model. Oftentimes, some coordinates are close to being normally distributed and the corresponding skewness parameters are unnecessary. Forward and backward selection procedures are adopted to eliminate such parameters and improve the model efficiency. These algorithms also prevent model-overfitting and conduct diagnostics with fitted skewness parameters. If underlying data groups are normally distributed, the selection procedures produce Gaussian mixture models.

The selection is based on the Bayesian information criterion (BIC) (Schwarz, 1978), which is the most commonly used criterion in finite mixture modeling (McLachlan and Peel, 2000). The smaller BIC is, the better fit provided by a mixture is. The forward selection procedure starts from the Gaussian mixture model and adds one $\lambda_{k j}$ at a time until no improvement in BIC value can be obtained. The produced model is called Manly forward model (denoted as Manly F in this paper) with the details of the method outlined in Algorithm 1. The backward model selection algorithm given in Algorithm 2 works in the opposite direction. It starts with the full Manly mixture and drops one skewness parameter $\lambda_{k j}$ at a time until no lower BIC can be reached. The obtained model is called

Data: $\boldsymbol{X}_{1}, \ldots, \boldsymbol{X}_{n}$

Result: estimated model parameters by Manly forward model

Initialization: Gaussian mixture model

while the current model $\boldsymbol{M}_{\text {current }}$ has not reached the full Manly mixture model do

1. find all zero skewness parameters in the current model $\boldsymbol{M}_{\text {current }}, \lambda_{1}, \ldots, \lambda_{t}$;

2. construct new models $\boldsymbol{M}_{n e w, 1}, \ldots, \boldsymbol{M}_{n e w, t}$ to compare with;

3. $\boldsymbol{M}_{n e w, j}$ sets the previous nonzero $K \times p-t$ skewness parameters and $\lambda_{j}$ to be non-zero;

4. call function Manly.EM() to run the EM algorithm for each new model;

5. initialize with the parameters of model $\boldsymbol{M}_{\text {current }}$ to speed the algorithm;

if at least one new model has lower BIC than the original model $\boldsymbol{M}_{\text {current }}$ then

find the smallest BIC among the new models;

the corresponding new model $\boldsymbol{M}_{\text {new }}$ is selected and let $\boldsymbol{M}_{\text {current }} \leftarrow \boldsymbol{M}_{\text {new }}$.

else

break;

the current model $\boldsymbol{M}_{\text {current }}$ is the final solution reached by Manly forward algorithm.

end

end

Algorithm 1: Manly forward selection algorithm. 
Data: $\boldsymbol{X}_{1}, \ldots, \boldsymbol{X}_{n}$

Result: estimated model parameters by Manly backward model

Initialization: full Manly mixture model $\boldsymbol{M}_{\text {full }}$ with $K \times p$ non-zero skewness parameters

while the current model $\boldsymbol{M}_{\text {current }}$ has not reached Gaussian mixture model do

1. find all non-zero skewness parameters in the current model $\boldsymbol{M}_{\text {current }}, \lambda_{1}, \ldots, \lambda_{s}$;

2. construct new models $\boldsymbol{M}_{n e w, 1}, \ldots, \boldsymbol{M}_{n e w, s}$ to compare with;

3. $\boldsymbol{M}_{n e w, j}$ sets the previous $K \times p-s$ skewness parameters and $\lambda_{j}$ to be zero;

4. call function Manly.EM() to run the EM algorithm for each new model;

5. initialize with the parameters of model $\boldsymbol{M}_{\text {current }}$ to speed the algorithm;

if at least one new model has lower BIC than the original model $\boldsymbol{M}_{\text {current }}$ then find the smallest BIC among the new models; the corresponding new model $\boldsymbol{M}_{\text {new }}$ is selected and let $\boldsymbol{M}_{\text {current }} \leftarrow \boldsymbol{M}_{\text {new }}$.

else

break;

the current model $\boldsymbol{M}_{\text {current }}$ is the final solution reached by Manly backward algorithm.

end

end

Algorithm 2: Manly backward selection algorithm.

the Manly backward model (Manly B). The selection algorithms are available in ManlyMix through setting method = "forward" or method = "backward" in the Manly.select () function.

\section{Manly K-means clustering}

Manly K-means clustering is constructed based on the classification EM (CEM) algorithm (Celeux and Govaert, 1992), which is a modification of the EM algorithm with an additional classification step. This step involves the Bayesian decision rule (i.e., $z_{i}^{(s)}=\operatorname{argmax}_{k} \pi_{i k}^{(s)}$ ) introduced immediately after the E-step.

It can be noticed that the traditional $K$-means algorithm is equivalent to the CEM algorithm based on the mixture model provided by

$$
g(x ; \Psi)=\frac{1}{K} \sum_{k=1}^{K} \phi\left(x ; \boldsymbol{\mu}_{k}, \sigma^{2} \boldsymbol{I}\right) .
$$

The model underlying the traditional $K$-means imposes very restrictive assumptions of the homoscedasticity and spherical structure of components. We alleviate these assumptions by allowing each component to have the covariance matrix $\sigma_{k}^{2} I$ and applying Manly transformation to the data. These changes result in the model given by

$$
g(x ; \Psi)=\frac{1}{K} \sum_{k=1}^{K} \phi\left(\mathcal{M}\left(x ; \lambda_{k}\right) ; \boldsymbol{\mu}_{k}, \sigma_{k}^{2} \boldsymbol{I}\right) \exp \left\{\boldsymbol{\lambda}_{k}^{T} \boldsymbol{x}\right\} .
$$

Following the same procedure as the Manly mixture EM algorithm, each $\lambda_{k}$ can be obtained separately by straightforward numeric optimization of the function $\tilde{Q}_{k}$ written as

$$
\begin{aligned}
\tilde{Q}_{k}\left(\boldsymbol{\lambda}_{k} \mid \Psi^{(s-1)}\right)= & -\frac{p n_{k}^{(s)}}{2} \log \left\{\sum_{i=1}^{n} \xi_{i k}^{(s)}\left(n_{k}^{(s)} \mathcal{M}\left(\boldsymbol{x}_{i} ; \boldsymbol{\lambda}_{k}\right)-\sum_{j=1}^{n} \xi_{j k}^{(s)} \mathcal{M}\left(\boldsymbol{x}_{j} ; \boldsymbol{\lambda}_{k}\right)\right)^{T}\right. \\
& \left.\times\left(n_{k}^{(s)} \mathcal{M}\left(\boldsymbol{x}_{i} ; \boldsymbol{\lambda}_{k}\right)-\sum_{j=1}^{n} \xi_{j k}^{(s)} \mathcal{M}\left(\boldsymbol{x}_{j} ; \boldsymbol{\lambda}_{k}\right)\right)\right\}+\lambda_{k}^{T} \sum_{i=1}^{n} \xi_{i k}^{(s)} \boldsymbol{x}_{i}+\text { const },
\end{aligned}
$$

where fuzzy classifications $\pi_{i k}^{(s)}$ are replaced by hard assignments in the form of indicators $\xi_{i k}^{(s)}=$ $I\left(z_{i}^{(s)}=k\right)$. If $z_{i}^{(s)}=k$ holds true, $\xi_{i k}^{(s)}$ takes a value of 1 ; otherwise $\xi_{i k}^{(s)}$ is equal to 0 . The current size of the $k$ th cluster is $n_{k}^{(s)}=\sum_{i=1}^{n} \xi_{i k}^{(s)}$.

In this way, each step of the Manly $K$-means algorithm updates the partition and parameter 
estimates. The partition update is given by

$$
z_{i}^{(s)}=\underset{k}{\operatorname{argmin}}\left\{\left\|\mathcal{M}\left(\boldsymbol{x}_{i} ; \boldsymbol{\lambda}_{k}^{(s-1)}\right)-\boldsymbol{\mu}_{k}^{(s-1)}\right\|^{2} /\left(2\left(\sigma_{k}^{2}\right)^{(s-1)}\right)-\left(\boldsymbol{\lambda}_{k}^{(s-1)}\right)^{T} \boldsymbol{x}_{i}+\frac{p}{2} \log \left(\sigma_{k}^{2}\right)^{(s-1)}\right\},
$$

while the parameters are estimated through the following expressions:

$$
\begin{gathered}
\boldsymbol{\lambda}_{k}^{(s)}=\underset{\lambda_{k}}{\operatorname{argmax}} \tilde{Q}_{k}^{(s)}\left(\boldsymbol{\lambda}_{k}\right), \quad \boldsymbol{\mu}_{k}^{(s)}=\sum_{i=1}^{n} \xi_{i k}^{(s)} \mathcal{M}\left(\boldsymbol{x}_{i} ; \boldsymbol{\lambda}_{k}^{(s)}\right) / n_{k}^{(s)}, \quad \text { and } \\
\left(\sigma_{k}^{2}\right)^{(s)}=\sum_{i=1}^{n} \xi_{i k}^{(s)}\left(\mathcal{M}\left(\boldsymbol{x}_{i} ; \boldsymbol{\lambda}_{k}^{(s)}\right)-\boldsymbol{\mu}_{k}^{(s)}\right)^{T}\left(\mathcal{M}\left(\boldsymbol{x}_{i} ; \boldsymbol{\lambda}_{k}^{(s)}\right)-\boldsymbol{\mu}_{k}^{(s)}\right) /\left(p n_{k}^{(s)}\right) .
\end{gathered}
$$

The Manly K-means algorithm is incorporated in the R package ManlyMix through the function Manly.Kmeans (). It can be used when the number of data points in each cluster is about the same and the transformed clusters are close to being spherical. It shows faster performance as the inversion of potentially large covariance matrices is not needed.

\section{Package functionality and illustrative examples}

All functions available in the package ManlyMix are listed with brief descriptions in Table 2. In this section, we demonstrate the utility of each function through a synthetic dataset and the analysis of two real-life datasets: Iris (Anderson, 1935; Fisher, 1936) and AIS (Cook and Weisberg, 1994).

\begin{tabular}{ll}
\hline Function & Description \\
\hline Manly.EM() & Runs the EM algorithm for a Manly mixture model \\
Manly.select() & $\begin{array}{l}\text { Runs forward and backward selection methods for a Manly mixture } \\
\text { model }\end{array}$ \\
Manly.Kmeans() & Runs the Manly K-means clustering \\
Manly.overlap() & Estimates the overlap values for a Manly mixture \\
Manly.sim() & Simulates datasets from Manly mixture models \\
Manly.var() & Performs variability assessment of Manly mixture model parameter \\
Manly.plot() & estimates and returns confidence intervals \\
ClassAgree() & Constructs a plot to display model-fitting and clustering \\
Manly.model() & Serves as a wrapper function for Manly mixture modeling \\
\hline
\end{tabular}

Table 2: Summary of functions implemented in ManlyMix.

\section{Illustrative example 1}

In this subsection, a Manly mixture is constructed with user-specified parameters. The overlap values of this mixture is estimated through function Manly. overlap(). Then function Manly. sim() simulates a dataset from the mixture along with a true membership vector.

\section{Step a: Mixture specification}

Now we demonstrate the procedure to construct a Manly mixture step by step. First, the user need to specify the number of components (assigned to $K$ ) and variables (assigned to $p$ ). In this case, we have a three-component bivariate mixture.

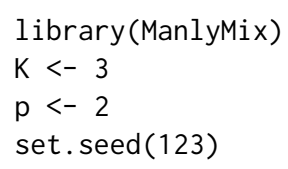

If the mixture probability density function of interest is written as

$$
\begin{aligned}
& g(\boldsymbol{x})=0.25 e^{0.2 x_{1}+0.25 x_{2}} \phi\left(\left(\begin{array}{c}
\frac{e^{0.2 x_{1}-1}}{0.2} \\
\frac{e^{0.25 x_{2}}-1}{0.25}
\end{array}\right) ;\left(\begin{array}{c}
4.5 \\
7
\end{array}\right),\left(\begin{array}{cc}
0.4 & 0 \\
0 & 0.4
\end{array}\right)\right) \\
& +0.3 e^{0.5 x_{1}+0.35 x_{2}} \phi\left(\left(\begin{array}{c}
\frac{e^{0.5 x_{1}}-1}{0.5} \\
\frac{e^{0.35 x_{2}}-1}{0.35}
\end{array}\right) ;\left(\begin{array}{l}
4 \\
8
\end{array}\right),\left(\begin{array}{cc}
1 & -0.2 \\
-0.2 & 0.6
\end{array}\right)\right) \\
& +0.45 e^{0.3 x_{1}+0.4 x_{2}} \phi\left(\left(\begin{array}{c}
\frac{0.3 e^{x_{1}}-1}{0.3} \\
\frac{e^{0.4 x_{2}}-1}{0.4}
\end{array}\right) ;\left(\begin{array}{c}
5 \\
5.5
\end{array}\right),\left(\begin{array}{cc}
2 & -1 \\
-1 & 2
\end{array}\right)\right) \text {, }
\end{aligned}
$$


we construct the mixture by assigning the model parameter values to la (matrix input of size $K \times p$ ), tau (vector input of length $K$ ), Mu (matrix input of size $K \times p$ ) and S (array input of dimensionality $p \times p \times K)$, respectively.

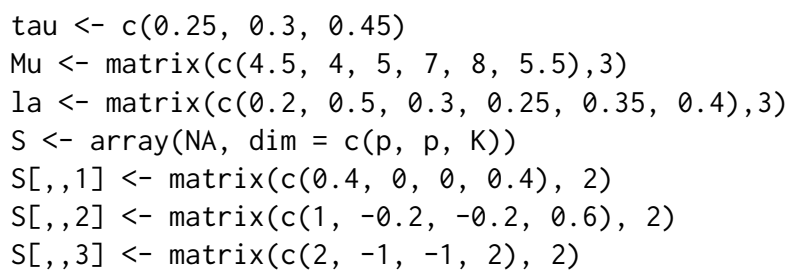

\section{Step b: Overlap assessment}

It is desirable to be capable of understanding the degree of interaction among mixing components to assess clustering complexity. Function Manly.overlap(), employing the measure of pairwise overlap, is implemented for this purpose. It has the following syntax:

$$
\text { Manly.overlap(tau, Mu, S, la, } \mathrm{N}=1000)
$$

with arguments $\mathrm{la}$, tau, Mu, S and $\mathrm{N}$. Here, $\mathrm{N}$ represents the number of samples simulated from the given mixture for pairwise overlap estimation. The larger $N$ is, the more precise the calculation is. By default, 1000 samples are employed. Four objects are returned by the function, including the misclassification probability matrix \$OmegaMap, pairwise overlap \$OverlapMap, average mixture overlap \$BarOmega, and maximum mixture overlap \$MaxOmega. Here, element \$OmegaMap $[\mathrm{k} 2, \mathrm{k} 1]$ corresponds to $\omega_{k_{1} \mid k_{2}}$ in Equation 7. In this case, for example, $\omega_{3 \mid 2}=0.046$ means that a random variable coming from the second component has approximate probability of 0.046 to be misclassified to group 3. $\omega_{3 \mid 3}=0.933$ represents the probability that a point belonging to group 3 is correctly assigned to this group. Each row of \$OmegaMap sums up to 1. Then, pairwise overlaps $\omega_{k_{1}, k_{2}}$ given in Equation 6 are provided in the \$Over lapMap. Among all pairwise overlaps $\left(\omega_{1,2}, \omega_{1,3}\right.$ and $\left.\omega_{2,3}\right), \omega_{2,3}$ yields the maximum value of 0.097 and produces $\$$ MaxOmega. The average of these three values, on the other hand, results in \$BarOmega being 0.08066667 .

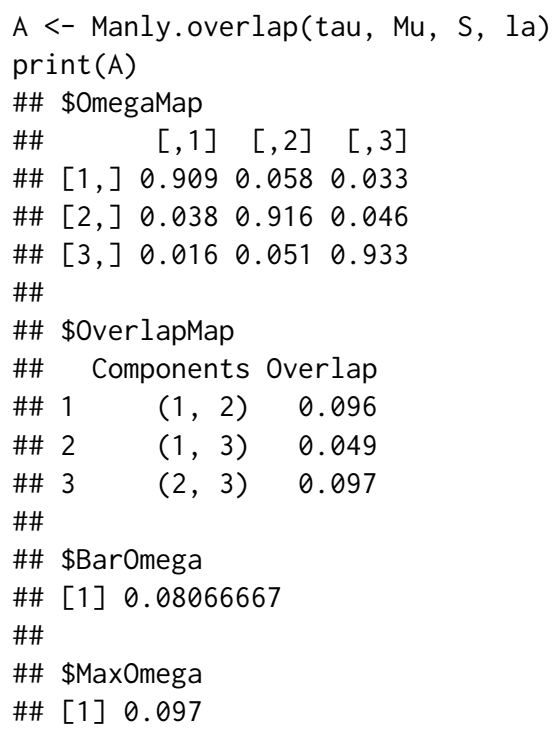

It can be seen that in the considered case, function Manly.overlap() calculates all characteristics based on the input of true model parameters. If parameters la, tau, Mu and S are estimated, Manly.overlap() provides estimates of misclassification probabilities and overlap values. As for high-dimensional data, we can not readily visually assess the interaction between data groups, such output helps approximate the proximity of clusters and discover properties associated with them.

\section{Step c: Data generation}

Function Manly.sim() simulates Manly mixture datasets based on user-specified model parameters. It employs the built-in $\mathrm{R}$ function rmultinom() for assigning data points to $K$ mixture components according to the mixing proportion $\tau_{k}$ 's. Then the function simulates normally distributed data points by function rnorm(). The covariance structures $\Sigma_{k}$ are applied to the data points before backtransforming them to Manly distributed components.

The Manly.sim() command has the following syntax: 
Manly.sim(n, la, tau, Mu, S)

The user can input $\mathrm{n}$ as the desired sample size. Here, a dataset of 30 observations is simulated from Equation 11 and data matrix $\$ \mathrm{X}$ as well as its true membership vector $\$ i d$ are returned.

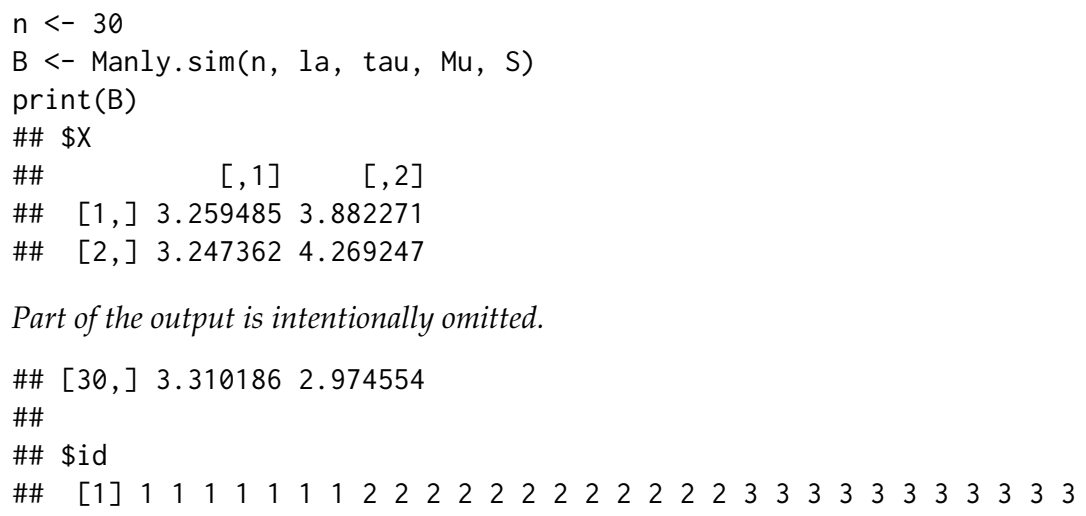

Part of the output is intentionally omitted.

\#\# [30,] 3.3101862 .974554

\#\#

\#\# \$id

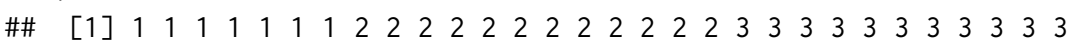

\section{Illustrative example 2: Iris dataset}

The Iris dataset (Anderson, 1935; Fisher, 1936) has 150 observations and 4 variables that represent sepal length, sepal width, petal length, and petal width. Three species, Iris setosa, Iris versicolor, and Iris virginica, have equal representation, consisting of 50 observations each. The function Manly. EM() fits a Manly mixture to the Iris dataset and 95\% confidence intervals of the model MLE are provided by Manly. var(). The Manly F and Manly B models are obtained by Manly. select(). The Manly K-means algorithm clusters the dataset through Manly. Kmeans().

\section{Step a: Data preparation}

Manly.EM() requires input of a matrix object $X$, where rows of $X$ represent $p$-variate observations. If $X$ is univariate data with vector input, it will be automatically transformed into a matrix of just one column. Thus, $\mathrm{X}$ has the dimensionality $n \times p$. In this case, we transform the Iris dataset into a matrix of dimensionality $150 \times 4$ and assign it to $X$.

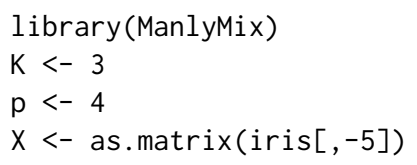

\section{Step b: Initialization of the EM algorithm}

Good initialization strategy of the EM algorithm is important to improve chances of finding a correct result. There are two ways for the user to initialize the Manly.EM() function. One is by means of providing the initial partition of the data id (vector input of length $n$ ) and skewness parameters la. Here, it needs to be noticed that the specification of la matrix serves as an indicator of whether the transformation is applied to a specific variable and component or not. For example, for the Iris dataset, assumes that all variables in all components enjoy normality except for the first variable in the first component, la needs to be set as la <-matrix $(c(0.1, \operatorname{rep}(0,11)), 3,4)$, with 0.1 (that can be any non-zero value) serving as the starting point in Nelder-Mead optimization. If no la is provided, the skewness parameters are all set equal to 0 and a Gaussian mixture model will be fitted. The other way of starting the algorithm is to enter initial model parameters, including la, tau, Mu, and S. The algorithm employs these parameters to compute the posterior probabilities in the first E-step.

Here, we adopt the first strategy. The initial partition of the Iris data is obtained by running the traditional $K$-means algorithm and specifying la is a matrix of size $3 \times 4$, with all elements set to a non-zero value of 0.1 .

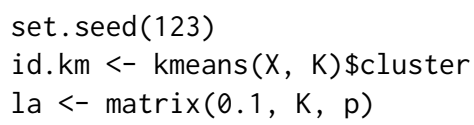

\section{Step c: EM algorithm for Manly mixture modeling}

Manly.EM() runs the EM algorithm for modeling based on Manly mixtures given in Equation 1. The command has the following syntax:

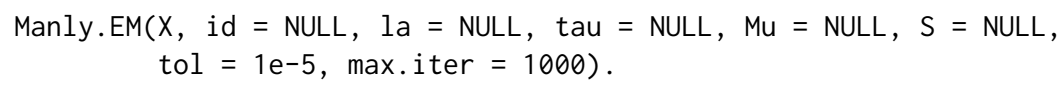


The parameters tol and iter correspond to the stopping rule for the EM algorithm. tol specifies the tolerance level of the EM algorithm. If the relative difference of the $Q$ function values from two consecutive steps is smaller than tol, the EM algorithm is terminated. By default, tol is set equal to $10^{-5}$. max. iter stands for the maximum number of iterations allowed for the EM algorithm. The default value of max. iter is 1000 . We fit the Iris dataset by both Gaussian mixture (assigned to object $\mathrm{G}$ ) and Manly mixture (assigned to object $\mathrm{M}$ ).

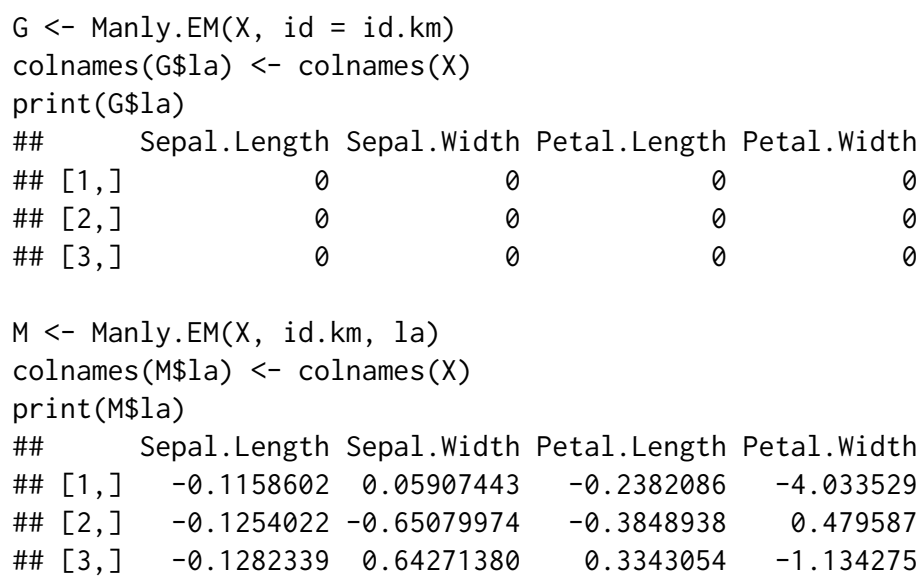

The estimated model parameters returned by the function Manly. EM() include \$la (matrix output of size $K \times p$ ), \$tau (vector output of length $K$ ), \$Mu (matrix output of size $K \times p$ ) and $\$ S$ (array output of dimensionality $p \times p \times K)$. They correspond to the parameters $\lambda_{k}, \tau_{k}, \boldsymbol{\mu}_{k}$ and $\boldsymbol{\Sigma}_{k}$ in Equation 3 and 4 , respectively. In this example, it can be observed that the returned $\$ 1$ a for the Gaussian mixture have all elements fixed at zero, while the Manly mixture has estimated the skewness parameters for each component and variable. For example, the skewness parameter associated with the sepal length variable of the first cluster is estimated to be -0.1158602 . It can be observed that most of the estimated parameters are relatively close to zero, which indicates approximate normality of the Iris data.

Some other parameters returned by Manly. $\operatorname{EM}()$ are the $n \times K$ matrix of posterior probabilities \$gamma calculated from Equation 2 in the last E-step and the membership vector \$id assigned by the Bayes decision rule in Equation 5. In this case, the output of \$id demonstrates the model-based clustering solution of the Iris dataset.

The characteristics of the fitted model are demonstrated in terms of the model log-likelihood \$11 and BIC \$bic. The number of iterations run by the EM algorithm until convergence is recorded through $\$$ iter. In this example, the EM algorithm reaches convergence after 13 iterations and the model BIC is 618.46. Finally, a dummy indicator $\$$ flag reports the validity of the fitted model, where 0 represents the successful convergence of the EM algorithm and 1 stands for the failure of convergence. A warning message is given if $\$ f l a g$ is equal to 1. It may happen when one cluster disappears or shrinks so that some parameter estimates are NA's. Such issue is related to spurious solutions (McLachlan and Peel, 2000) where one or more components model a local pattern in data rather than a systematic one.

\section{Step d: Variability assessment of Manly mixture model}

Variability assessment of the model parameters allows practitioners to study the specific nature of the fitted model as well as detected clustering solutions. We provide the user with function Manly. $\operatorname{var}()$, which calculates the inverse of the empirical observed information matrix given in Equation 8 and returns the variance-covariance matrix of the estimated MLE from Manly. EM() function. It also outputs the confidence intervals of each parameter.

The command has the following syntax:

$$
\text { Manly. } \operatorname{var}(X, \operatorname{model}=\text { NULL, } \operatorname{conf} . C I=N U L L)
$$

$X$ represents the data matrix and model is the object of class "ManlyMix". conf.CI is user-specified confidence level, which needs to take a value between $\theta$ and 1 . Here by setting model $=M$, we take the MLE of the fitted Manly mixture obtained from step $\mathrm{c}$ and evaluate its variability. The number of unique model parameters is $K-1+2 K \times p+K \times p(p+1) / 2=56$ for the Iris dataset. conf. CI = 
0.95 calculates $95 \%$ confidence intervals for these 56 parameters. Thus Manly.var () function returns a $56 \times 56$ covariance matrix (assigned to $\mathrm{V}$ ) and 56 confidence intervals (assigned to CI).

result <- Manly. $\operatorname{var}(X$, model $=M$, conf. $C I=0.95)$

In the code output of $95 \%$ confidence intervals, the first column represents the point estimates of the 56 model parameters, while the second and third columns stand for the lower and upper bounds of confidence intervals, respectively.

\begin{tabular}{lrrrr}
\multicolumn{6}{l}{ print(result\$CI) } & & \\
$\# \#$ & Estimates & Lower & Upper \\
$\# \#$ & {$[1]$,} & 0.333333333 & 0.257887628 & 0.408779039 \\
$\# \#$ & {$[2]$,} & 0.264119102 & 0.175676489 & 0.352561716 \\
$\# \#$ & {$[3]$,} & 3.794594378 & -8.303528084 & 15.892716840
\end{tabular}

Part of the output is intentionally omitted.

$\begin{array}{rrrr}\text { \#\# }[54,] & 0.642713799 & -0.407079526 & 1.692507125 \\ \text { \#\# [55, ] } & 0.334305435 & -0.128841410 & 0.797452280 \\ \text { \#\# [56, ] } & -1.134275340 & -2.079185136 & -0.189365544\end{array}$

\section{Step e: Forward and backward selection algorithms}

Step e targets detecting the normally distributed variables in Iris. Manly. select() provides the selection algorithm for eliminating unnecessary skewness parameters in $M \$ l a$. These skewness parameters are fixed to be equal to zero and the log-likelihood is maximized based on the rest of parameters. The use of the function is shown below:

$$
\begin{aligned}
& \text { Manly. } \operatorname{select}(X, \text { model, method, tol }=1 e-5, \text { max.iter }=1000, \\
& \text { silent }=\text { FALSE })
\end{aligned}
$$

The argument model is the initial model to start the selection procedure with. method is set to either "forward" or "backward" for the implementation of Algorithm 1 or Algorithm 2, respectively. The selection criterion for each step is based on \$bic values obtained from all candidate models that are of class "ManlyMix". silent is an argument that controls the code output. By default, silent provides the steps of selection and BIC values for all candidate models. Thus, the user can monitor the selection procedures. The output can be turned off by setting silent $=$ TRUE. We first discuss the implementation of the forward selection on the Iris dataset. The algorithm is initialized by the Gaussian mixture model G obtained in step c.

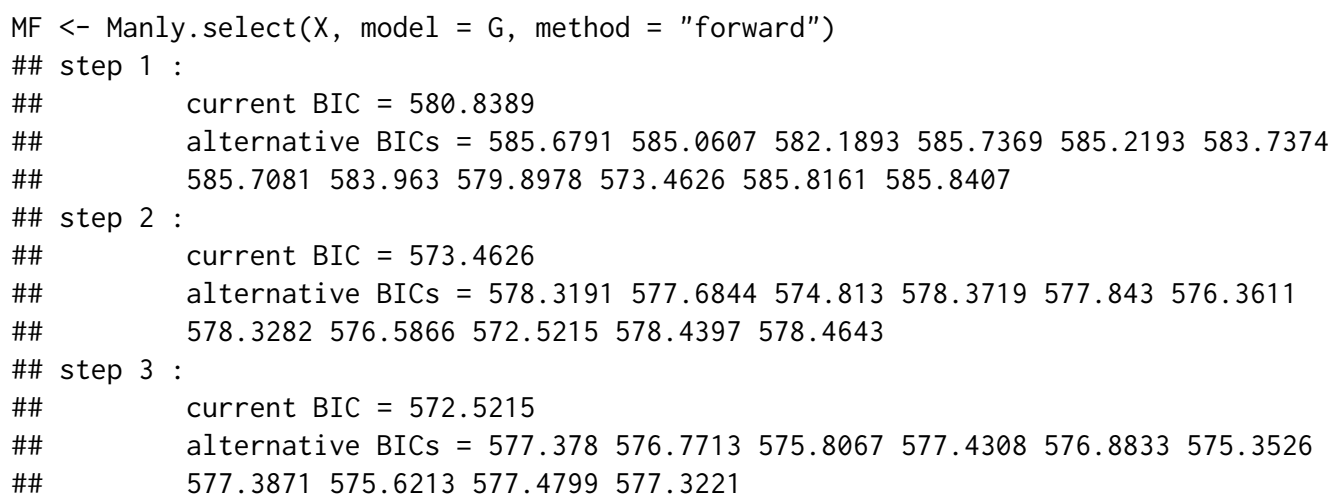

The forward selection takes three steps for the algorithm to find the best model (assigned to MF). In step 3, there is no alternative BIC value that is smaller than the current model BIC, so the forward selection algorithm stops searching over non-zero $\lambda_{k j}$ 's. Compared to the Gaussian mixture fit, Manly F model improves by 8 in BIC value.

On the contrary, the backward selection starts with the full Manly mixture M and drops one skewness parameter at a time.

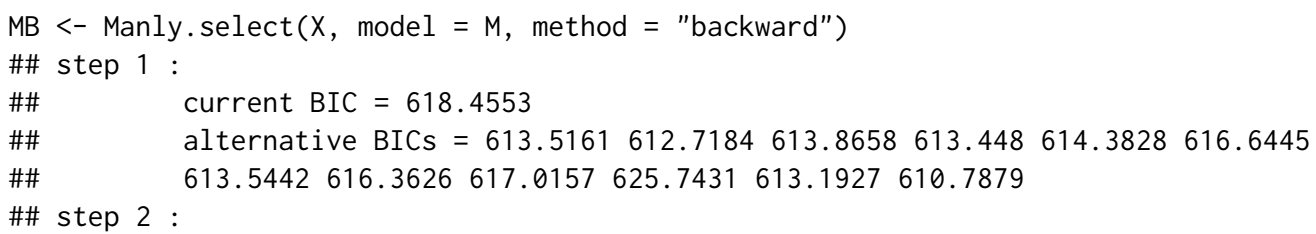




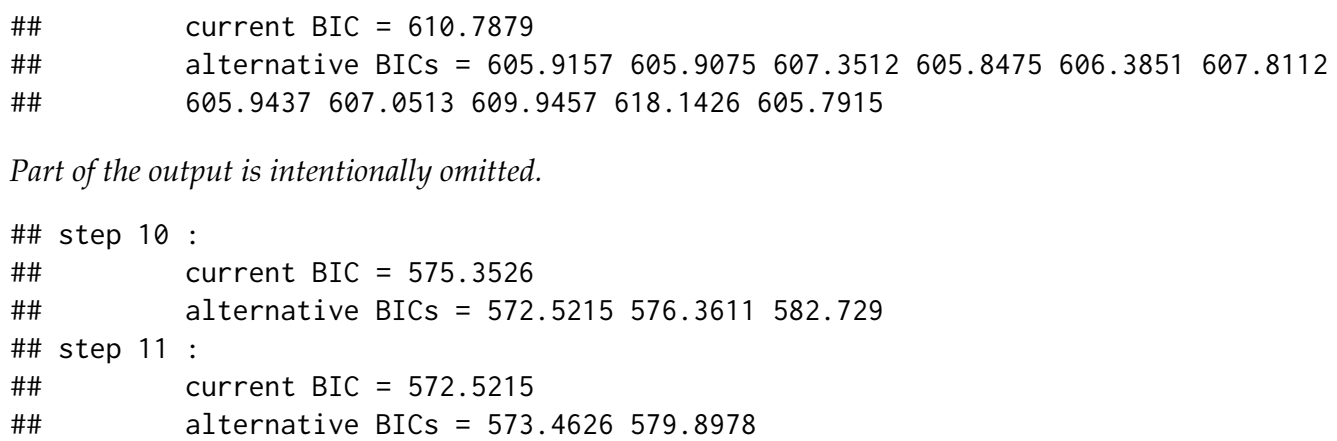

After 11 steps, the backward selection produces the Manly B model, which enjoys the same BIC value as the Manly F model.

\section{Step f: Diagnostics}

The skewness parameters of the Manly F and Manly B models are investigated in the following example. It is observed that the forward selection adopts only two $\lambda_{k j}$ 's in the model. They correspond to the petal width variable of the first species and the petal length variable of the third one. For all other components and variables, the data appear to be nearly normally distributed. The same two skewness parameters are found by the backward selection. It is worth mentioning, however, that Manly F and Manly B models can produce different results.

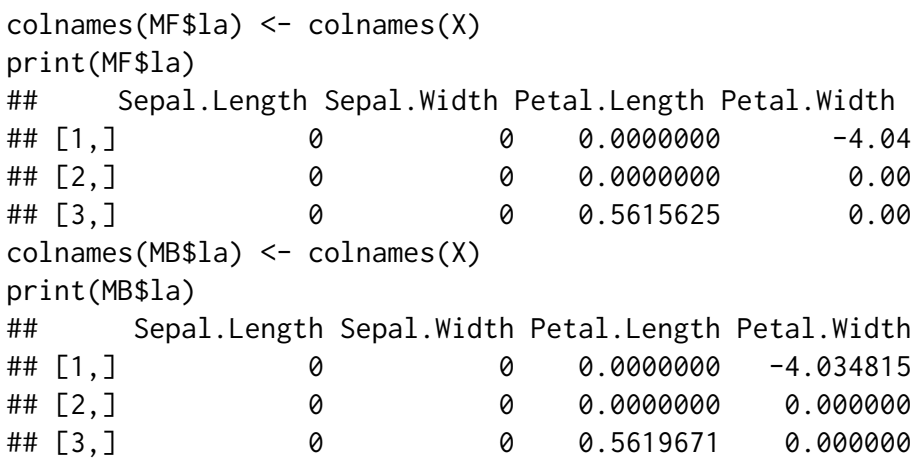

\section{Step g: Manly K-means algorithm}

The Manly K-means algorithm written in Equation 9 is implemented in function Manly.Kmeans(), which has the following syntax:

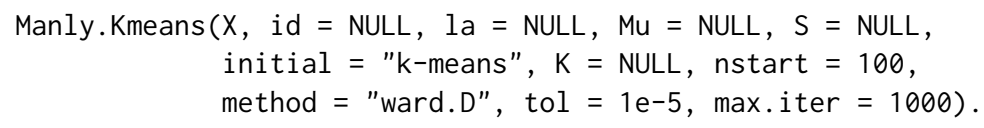

Manly.Kmeans() has most of the arguments and returned values the same as those of the function Manly.EM(). As the Manly K-means algorithm assumes that all clusters are of the same size, the mixing proportions tau are not needed in this function. $S$ is a vector of length $K$ that represents variance within each cluster, as the transformed data groups are assumed to be spherical. The parameters returned by the function $\$ \mathrm{la}$, $\$ \mathrm{Mu}$, and $\$ \mathrm{~S}$ correspond to $\lambda_{k}, \boldsymbol{\mu}_{k}$ and $\sigma_{k}^{2}$ given in Equation 10. The log-likelihood and BIC values are not provided since the parameter estimates are not MLE's. Manly. Kmeans() has several initialization choices: (1) by providing id and la; (2) by providing la, Mu, and S; (3) by specifying the number of clusters $\mathrm{K}$ and letting initial = "k-means"; It takes the default traditional K-means clustering result and passes it into the CEM algorithm; (4) by specifying the number of clusters $\mathrm{K}$ and letting initial = "hierarchical"; It adopts the hierarchical clustering solution as the initial dataset partition. nstart is responsible for controlling the number of random starts tried in initialization choice (3) with a default value equal to 100. method sets the linkage method in initialization choice (4) with a default of method = "ward. D", which represents the Ward's linkage (Ward, 1963). Here, the initialization choice of Manly. Kmeans() is (1), which is the same as that of Manly. EM().

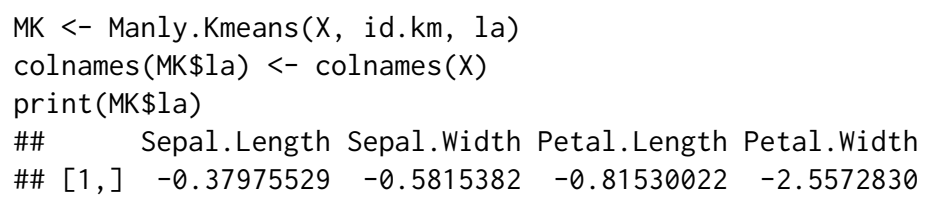




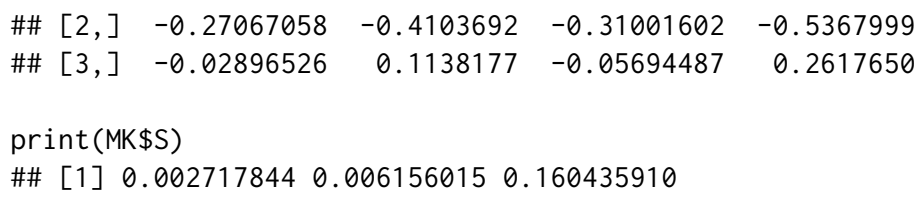

\section{Illustrative example 3: AIS dataset}

In this subsection, dataset AIS (Cook and Weisberg, 1994) is studied for illustrative purposes. The Australian Institute of Sports (AIS) dataset was first introduced by Cook and Weisberg (1994). It contains information collected from 202 athletes, among which 100 are females and 102 are males. There are 13 variables, including the gender, sport kind and 11 numeric measurements of the athletes. We adopt the same variables and analysis as Lee and McLachlan (2013). The goal of the analysis is to cluster the athletes into two groups: males and females by constructing models based on three measurements: the body mass index ("BMI"), lean body mass ("LBM"), and the percentage of body fat ("Bfat"). Function ClassAgree() compares the estimated and true partitions. Function Manly.plot() is introduced for visual analysis of Manly mixture fitted results.

\section{Step a: model fit}

The AIS dataset is analyzed by six mixture models: the traditional $K$-means (kmeans ()), Manly Kmeans (Manly.Kmeans()), Gaussian mixture model (Manly.EM()), Manly mixture model (Manly.EM()), Manly forward model and Manly backward model (both available through Manly. select()).

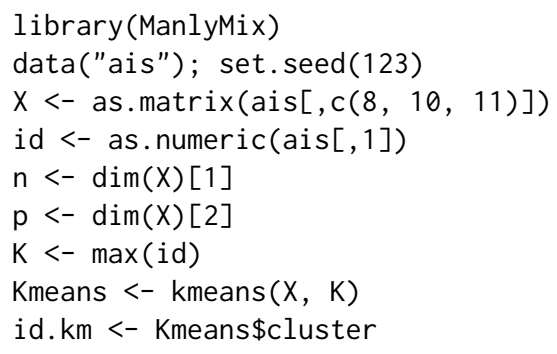

By running the following code, we not only obtain the fitted models, but also test the package from different aspects. The number of parameters in the models are 7 (K-means), 19 (Gaussian), 25 (Manly), 23 (Manly F), 22 (Manly B) and 14 (Manly K-means). The computing times are 0.001, 0.004, $0.083,0.8,1.143,0.024$, respectively. These results are rather efficient compared to those from other packages (see Appendix).

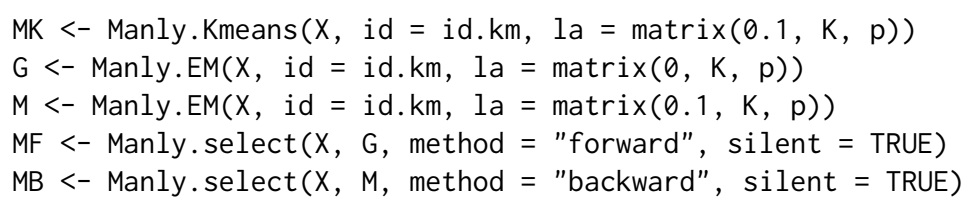

Now we consider the fitted model parameters to perform a comprehensive analysis and diagnostics of the AIS dataset. From the following output, it is observed that the Manly F model drops two skewness parameters from the full Manly mixture model while Manly B drops three. This yields the conclusion that the "Bfat" variable in the first group and "LBM" variable in the second one are close to be normal. Through the one-to-one correspondence between skewness parameters and dataset variables, ManlyMix is proved to be particularly useful for model variable diagnostics.

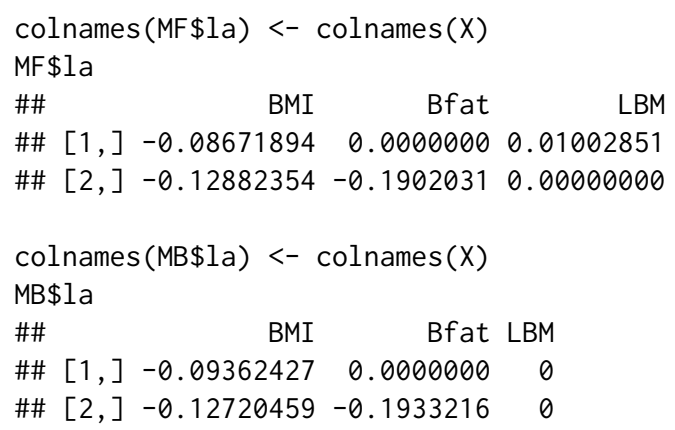

BIC values for the four models are 3595.35 (Gaussian), 3543.00 (Manly), 3538.42 (Manly F) and 3533.63 (Manly B). It shows considerable improvement in terms of BIC from Manly mixture models. 
They provide better fits for the data, among which Manly backward is the best model selected according to BIC.

\section{Step b: classification table}

Classification results from the six models are compared using function ClassAgree() in step b. Function ClassAgree() adopts input of both the estimated and true id vectors with the following syntax:

ClassAgree(est.id, trueid)

ClassAgree() permutes the partition labels to achieve the lowest number of misclassifications. Then, based on the switched labels, it returns the confusion matrix and number of misclassifications. In the analysis of the AIS dataset, the following output is produced by ClassAgree().

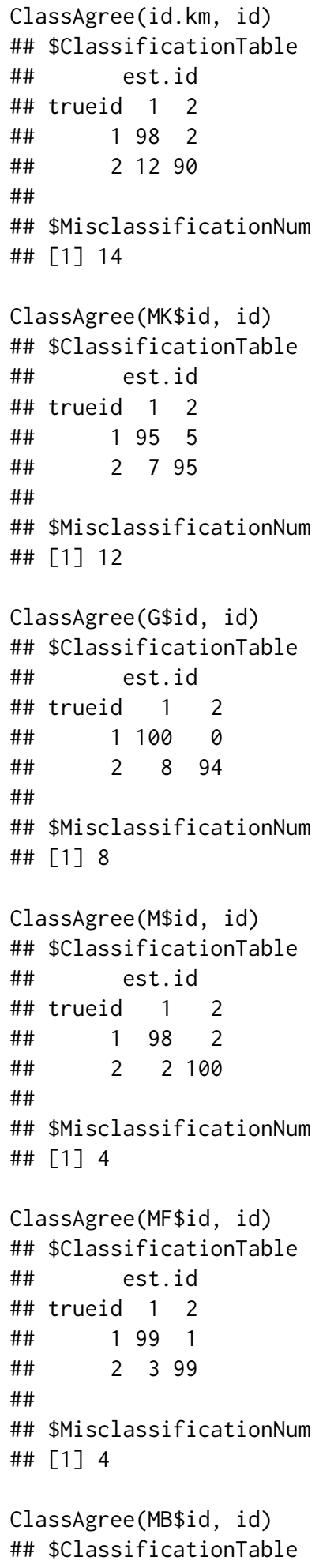




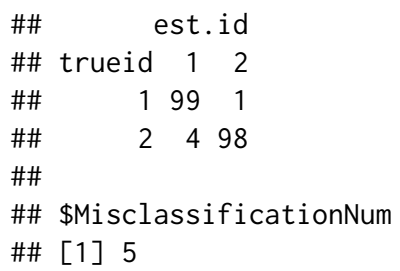

Rows and columns represent the true and estimated partitions, respectively. The diagonal and offdiagonal elements in the table correspond to correct and incorrect classifications, respectively. The lowest number of misclassifications (4 misclassifications) is obtained by the Manly mixture and Manly forward models. One worth-mentioning fact is that these two models enjoy the clustering solution as good as the unrestricted skew- $t$ mixture, which is reported to be the best model by Lee and McLachlan (2013). The Manly backward model comes second with 5 misclassifications. The remaining three models, traditional $\mathrm{K}$-means, Manly $\mathrm{K}$-means and Gaussian mixture model show worse performance.

\section{Step c: visualization tool}

In order to investigate the behavior of each model, contour plots with classified data points need to be analyzed. Manly.plot() allows conducting the visual analysis of a dataset fitted by Manly mixture model. The command has the following syntax:

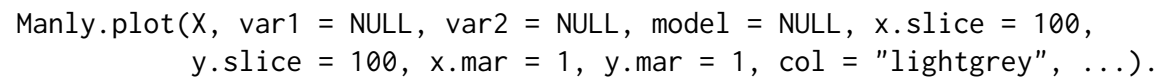

If both var 1 and var 2 are provided, they represent variables on the $X$-axis and $Y$-axis of a contour plot, respectively. Argument model is the object of class "ManlyMix". The parameters of model object are used to calculate the density and draw contour lines. The estimated membership vector model\$id is reflected through different colors. $x$.slice and $y$.slice options control the number of grid points for which a density is calculated. The larger these two values are, the more grid values are considered. Thus, the contour lines look smoother. $x$.mar and y.mar specify plot margins. The parameter col specifies the color of contour lines with the default color being light grey. Other variables in the built-in $\mathrm{R}$ function contour () can also be used as specified. On the other hand, if only var 1 is provided, a density plot of this variable is constructed. $x$. slice and x. mar have the same functionality as those in the contour plot. The parameter col stands for density line color with the default being light grey. ... allows other arguments from the built-in R function hist() to be passed.

In this case, we conduct the same analysis as that in Lee and McLachlan (2013) and adopt the two variables "LBM" and "Bfat" for constructing contour plots. The margins of the plots are set to be 3 on the $X$-axis and 13 on the $Y$-axis. The light grey contour lines have width equal to 3.2. Labels and axes are suppressed. The function is first applied to the four fitted models Gaussian mixture, Manly mixture, Manly F and Manly B in step a.

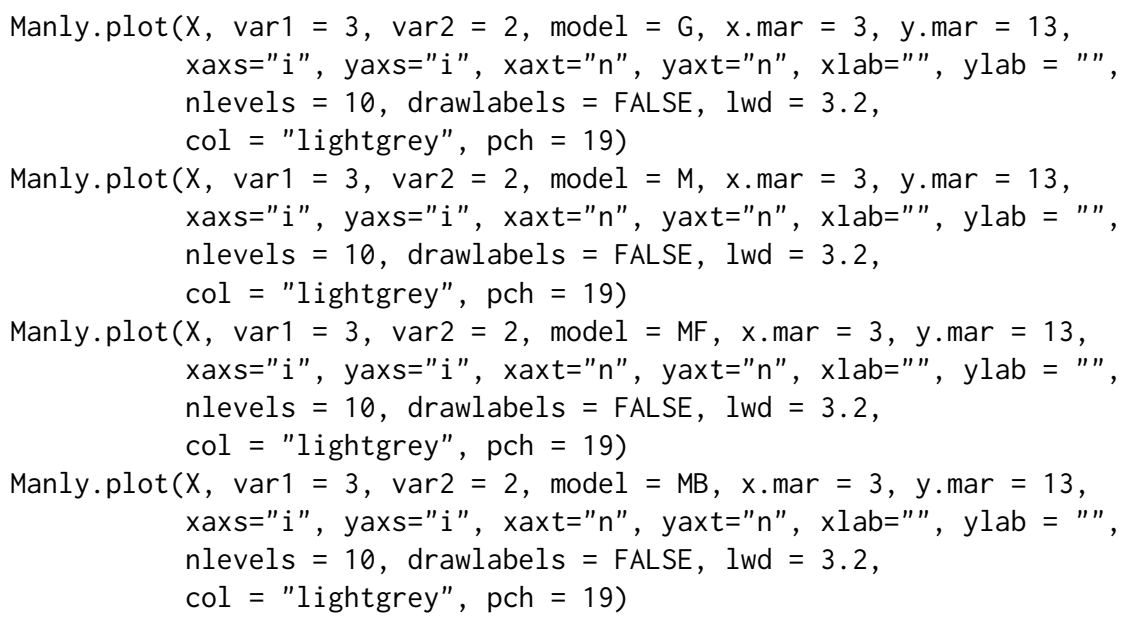

Function Manly.plot() enjoys sufficient flexibility to adopt other parsimonious models. Parameters obtained by traditional $\mathrm{K}$-means and Manly $\mathrm{K}$-means can be adjusted according to the object of class "ManlyMix" so that \$id, \$tau, \$Mu, \$la, \$S are extracted in their correct forms.

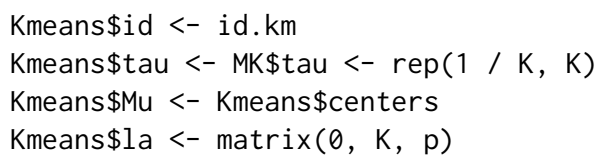




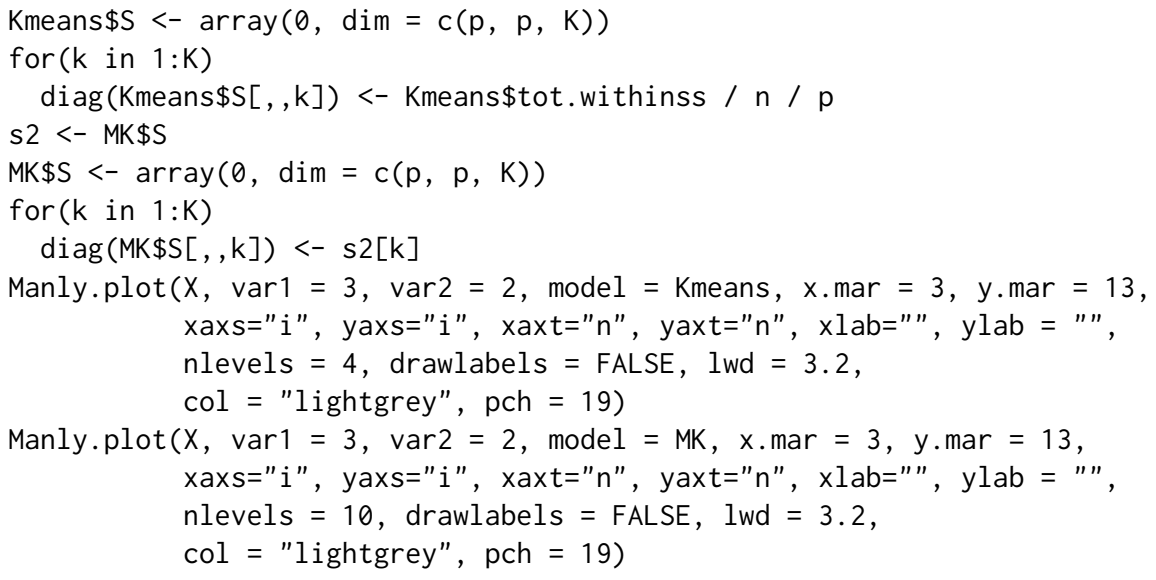

Figure 1 combines all output plots from Manly.plot().

It can be observed that the two components have a slight overlap, so the clustering problem is not over-complicated. However, the red cluster is highly skewed and has a heavy tail. This imposes difficulties for the traditional K-means, Manly K-means, and Gaussian mixture model. Manly mixture model shows great flexibility and captures the skewness pattern in both components. Manly forward drops the skewness parameters associated with variable "Bfat" in the female cluster (black component) and "LBM" in the male group (red component). Manly backward drops both skewness parameters that correspond to the female cluster and uses a black ellipsoid. It also drops the "LBM" variable in the male cluster (red component). The above results reveal the applicability and effectiveness of function Manly.plot() on real-life datasets.

\section{Alternative coding d: wrapper function}

Wrapper function Manly. model() enables practitioners to run analysis in a simple and convenient way. The function has the following syntax:

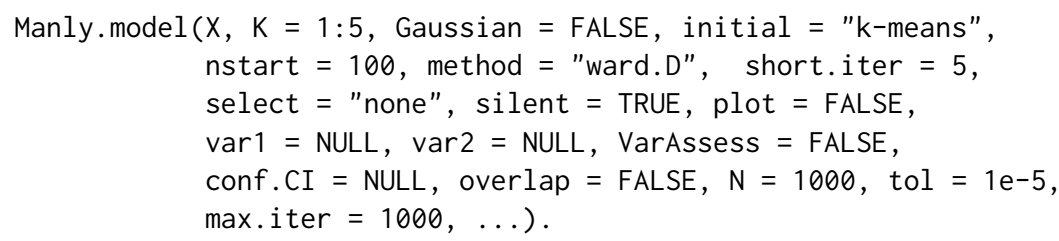

Argument $\mathrm{K}$ is an integer vector providing the numbers of clusters to be tested for the data. The default setting tests 1, 2, 3, 4, or 5 clusters. It calls the Manly. EM() function to fit all five models. The one with the lowest BIC value is chosen to be the best model. Gaussian option specifies whether skewness parameters are adopted or not. If TRUE, Gaussian mixtures are fitted. With the default value being FALSE, it runs full Manly mixture models. initial specifies the initialization strategy used. It has three input options: (1) initial = "k-means" is the default initialization strategy, which passes the traditional $K$-means result into the EM algorithm as the initial partition. nstart is passed into the built-in R function kmeans for specifying the number of random starts (the default nstart $=100$ ); $(2)$ if initial = "hierarchical", the hierarchical clustering initialization is used. The linkage method is passed by method argument into $\mathrm{R}$ function hclust. The default is Ward's linkage; (3) if initial = "emEM", the emEM (Biernacki et al., 2003) initialization is run. Short runs of EM are conducted based on random starts and the one that corresponds to the highest log-likelihood is picked for running until convergence. nstart controls the number of random starts. The number of iterations for the short EM is specified by short. iter with a default value set to 5 iterations.

select argument has three input values: "none", "forward" and "backward". If select = "none", then the object returned by function Manly.EM() is adopted directly. If select = "forward", the Gaussian option is automatically adjusted to Gaussian = TRUE. It calls function Manly. select $(\ldots$, method = "forward") to improve the original Gaussian fit. On the other hand, if select = "backward", Gaussian option is automatically set to Gaussian = FALSE. The full Manly mixture is followed by the backward selection Manly. select (..., method = "backward"). silent argument controls the output in function Manly.select(). The default setting suppresses the output. plot determines whether Manly.plot () function is called or not. If plot $=$ TRUE, then Manly.plot () runs and arguments var 1 and var2 allow user to specify which variable(s) to plot. Argument VarAssess provides the option of using Manly. $\operatorname{var}()$ for variability assessment. Notice here that it only provides assessment for a full Manly mixture model. conf. CI specifies the confidence level of the confidence intervals returned. The overlap option, if specified to be TRUE, adopts the Manly. overlap() function and estimates pairwise overlap values for the returned model. $\mathrm{N}$ is the number of Monte Carlo simulations run in 

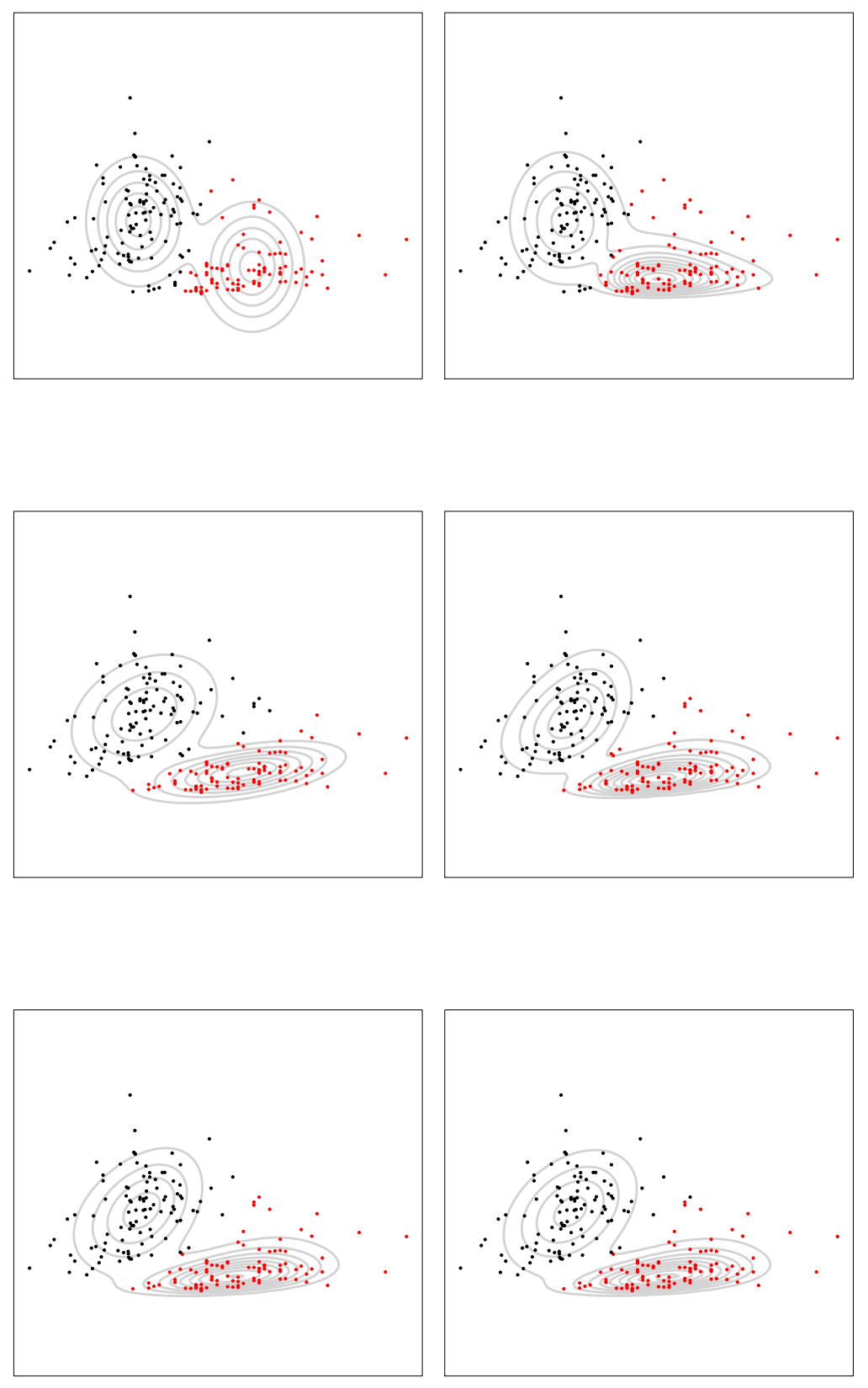

Figure 1: AIS dataset: fitted contour plots from function Manly.plot() based on the two variables "LBM" (X-axis) and "Bfat" (Y-axis). The model locations are: K-means (first row left), Manly K-means (first row right), Gaussian mixture (second row left), Manly mixture (second row right), Manly forward (third row left) and Manly backward (third row right). 
Manly. overlap().

Three objects are returned by function Manly. model(): \$model, \$VarAssess, and \$Overlap. \$model is the final model of class "ManlyMix" by Manly. EM() or Manly. select(). \$VarAssess returns the variance-covariance matrix and confidence intervals by Manly. var () function. \$Overlap returns the object by Manly. overlap().

For AIS dataset, suppose the user wants to obtain the Manly F or Manly B model and take a look at their contour plots. A compact version of the code is given by:

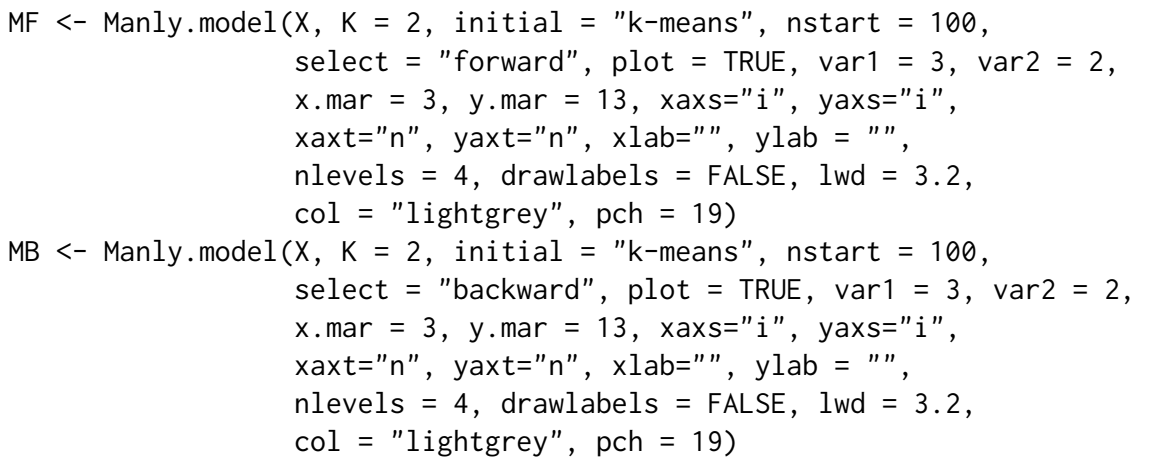

Here, MF \$model and MB\$model obtained are the same as those in step a. The contour plots are generated automatically and can be found in Figure 1.

\section{Alternative coding e: initialization with model parameters}

Functions Manly.EM() can take initial model parameters as initialization of the algorithm. It is especially useful for the emEM initialization. The practitioner can construct a large number of short EM runs, select the one with the highest log-likelihood and obtain its estimated parameters. Then, the EM algorithm initialized by these parameters is run until convergence. Here is a small example on the AIS dataset. 100 short EM algorithms run for 5 iterations each. As we can see, the obtained object $\mathrm{M}$ is the same as that from step a.

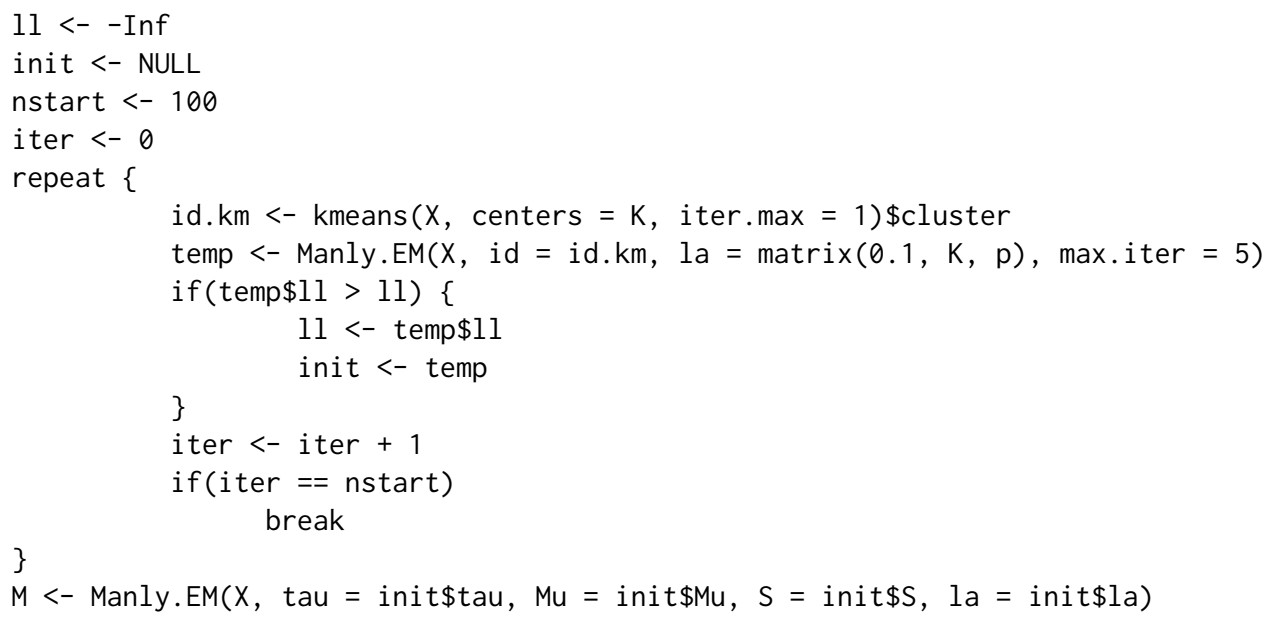

\section{Illustrative example 4: acidity dataset}

Since one reviewer is interested in seeing a showcase of a univariate Manly mixture, we illustrate its utility on the acidity dataset (Crawford, 1994). It provides the acidity measure of 155 lakes in the Northeastern United States. There are two clusters, but the true partition is unknown.

\section{Step a: model fit}

We run the following models: the traditional K-means (kmeans()), Manly K-means (Manly.Kmeans()), Gaussian mixture model (Manly. EM()), Manly mixture model (Manly. EM()), Manly forward model, and Manly backward model (both available through Manly. select()).

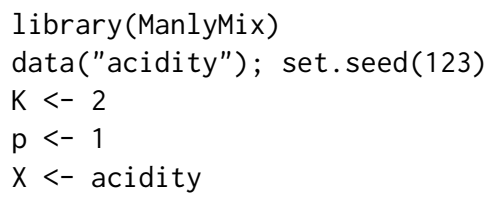


Kmeans <- $\operatorname{kmeans}(\mathrm{X}, \mathrm{K})$

id. $\mathrm{km}<-$ Kmeans\$cluster

MK <- Manly.Kmeans (X, id $=$ id. km, la $=\operatorname{matrix}(0.1, \mathrm{~K}, \mathrm{p}))$

$\mathrm{G}<-$ Manly.EM(X, id $=\mathrm{id} . \mathrm{km}, \mathrm{la}=\operatorname{matrix}(\theta, \mathrm{K}, \mathrm{p}))$

$M<-\operatorname{Manly} \cdot \operatorname{EM}(X, i d=i d . k m, l a=\operatorname{matrix}(0.1, K, \mathrm{p}))$

MF <- Manly.select $(X, G$, method = "forward", silent $=$ TRUE $)$

MB <- Manly.select $(X, M$, method = "backward", silent $=$ TRUE)

The model BIC values for Gaussian, Manly, Manly F and Manly B are 394.51, 389.84, 389.84 and 389.84, respectively. There is an indication of skewness as both the Manly F and Manly B models fail to drop any skewness parameters. The Manly models improve by 5 in the BIC value.

Step b: visualization tool

To visually assess the fit provided by all models, we use the command Manly.plot() with univariate input. The fitted density plots associated with histogram of the data are provided in Figure 2.

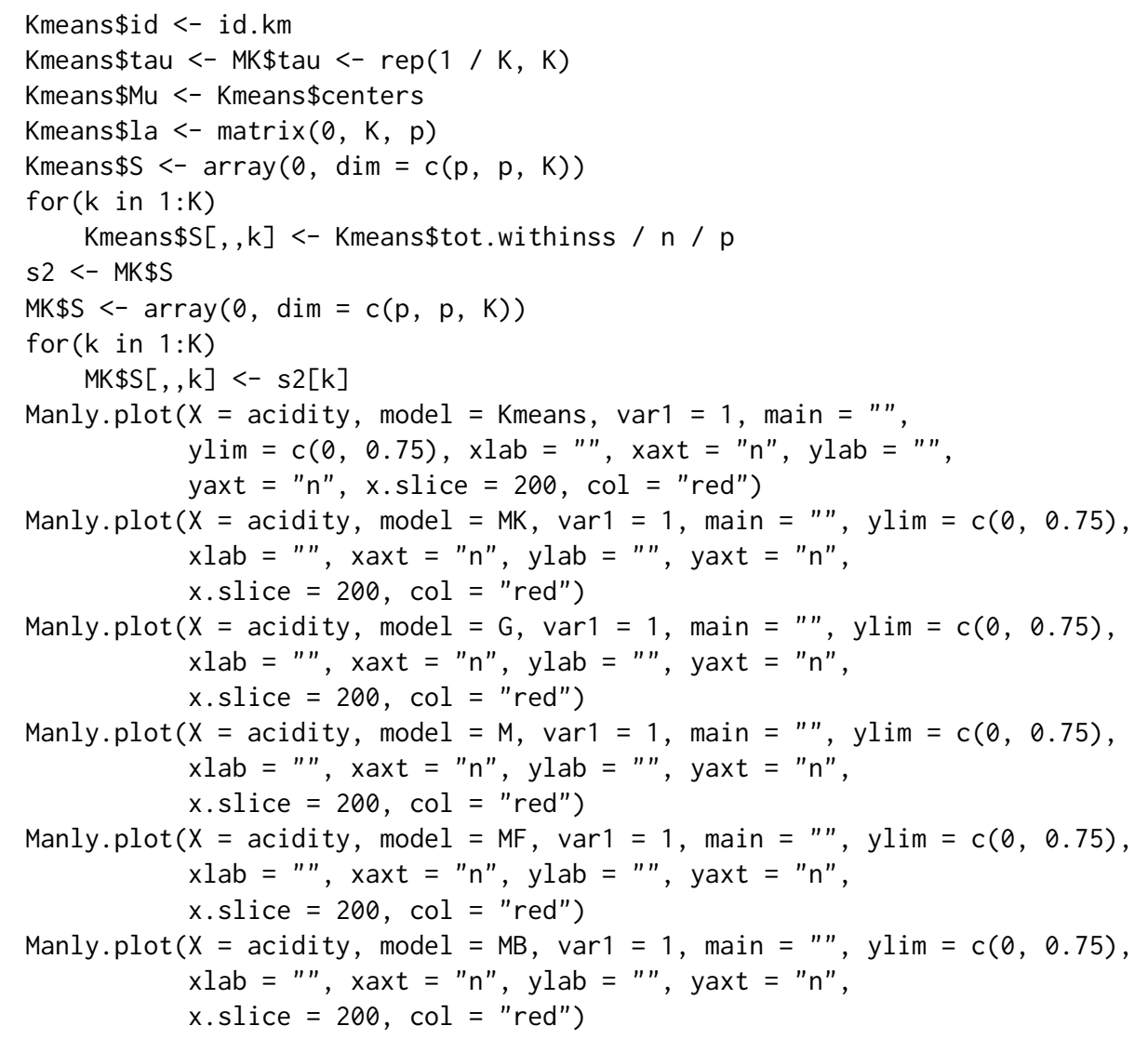

Manly models provide the most reasonable fit of the data. The first component is slightly skewed to the right and only the Manly models pick up the high density at its peak. The second component is slightly skewed to the left. The density fits provided by K-means and Manly K-means are insufficient due to the assumption of equal size components.

\section{Alternative coding c: wrapper function}

The wrapper function Manly. model() is capable of combining steps a and b in one command. The following code directly yields the Manly F or Manly B model:

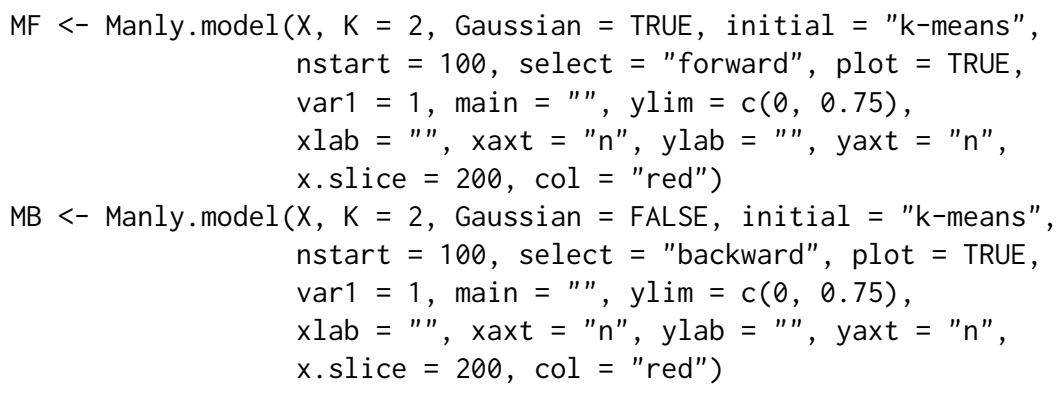

The Manly F and Manly B density plots given in Figure 2 are generated automatically. 

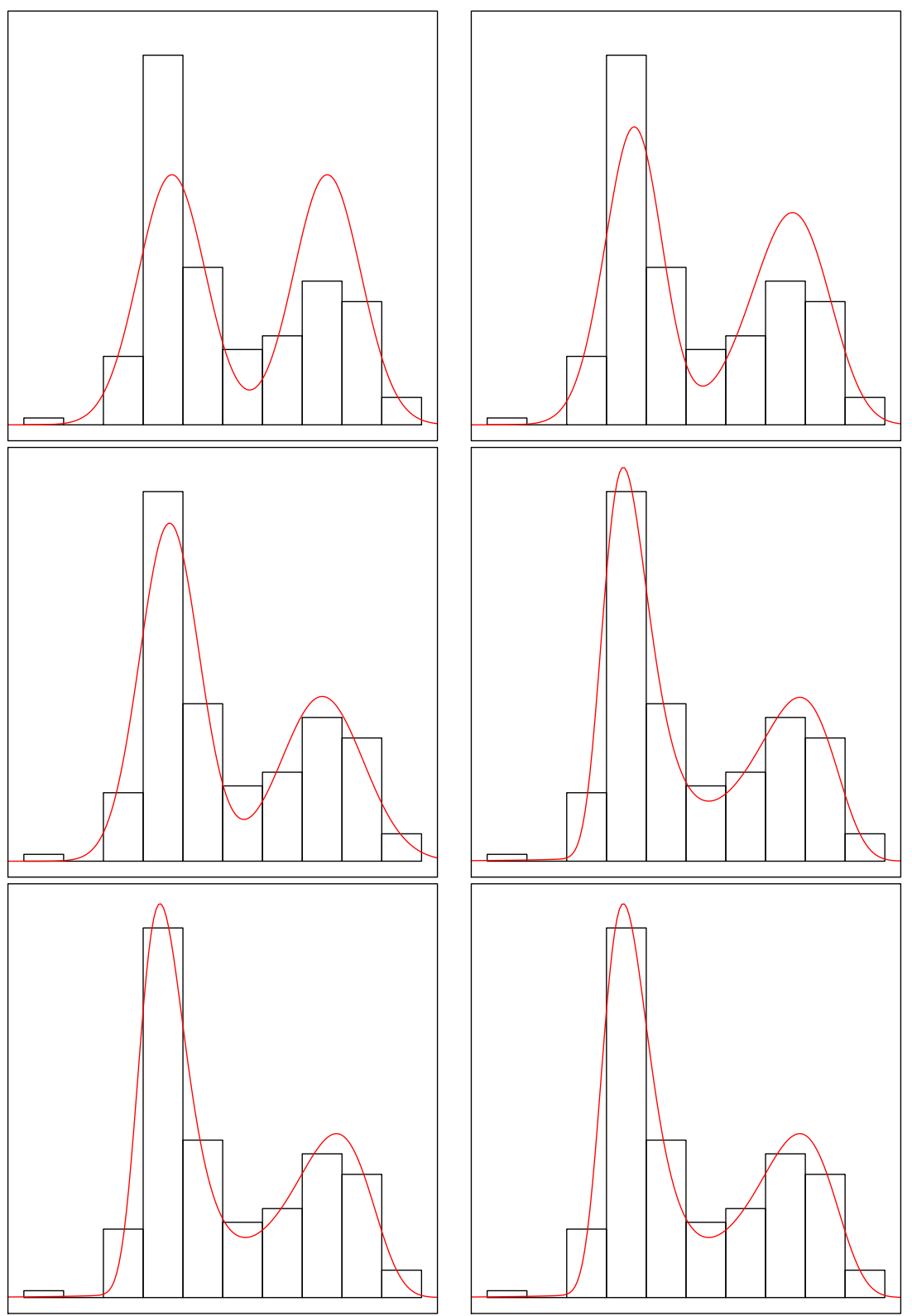

Figure 2: acidity dataset: fitted density plots from function Manly.plot (): K-means (first row left), Manly K-means (first row right), Gaussian mixture (second row left), Manly mixture (second row right), Manly forward (third row left) and Manly backward (third row right). 


\section{Demo examples}

For users who need further information about the package, we have constructed 16 demo examples listed in Table 3 that provide a comprehensive demonstration of ManlyMix capabilities. Among the examples, 11 of them are designed to demonstrate the capability and utility of each function and 5 of them run comprehensive analysis of classification datasets. Each demo can be accessed by its name and the users can reproduce themselves.

As an illustration of how these demos can be employed, the code of the first example can be approached through running the following code in $\mathrm{R}$.

library (ManlyMix)

demo(EMalgorithm1)

\begin{tabular}{ll}
\hline Function & Demo example(s) \\
\hline Manly.EM() & demo(EMalgorithm1), demo(EMalgorithm2) \\
Manly.select() & demo(ForwardSelection), demo(BackwardSelection) \\
Manly.Kmeans() & demo(ManlyKmeans1), demo(ManlyKmeans2) \\
Manly.overlap() & demo(Overlap) \\
Manly.sim() & demo(DataSimulation) \\
Manly.var() & demo(VarAssess) \\
Manly.plot() & demo(DensityPlot), demo(ContourPlot) \\
Comprehensive analysis & demo(utility), demo(ais), demo(seeds), demo(bankruptcy) \\
& demo(acidity) \\
\hline
\end{tabular}

Table 3: Summary of demo examples included in ManlyMix.

\section{Summary}

The R package ManlyMix is discussed and illustrated in detail. The provided functions enable practitioners to analyze heterogeneous data and conduct cluster analysis with Manly mixture models. The algorithms behind functions are introduced and explained carefully. Illustrative examples based on challenging real-life datasets are studied to demonstrate the usefulness and efficiency of the package. Promising results suggest that ManlyMix is not only a powerful package for clustering and classification, but also a diagnostic tool to investigate skewness and deviation from normality in data. Demo examples are provided for each function in ManlyMix for the users to study.

\section{Appendix}

The six competitors for mixture modeling of skewed data given in Table 1 are applied to the AIS dataset in Section 2.3.3, including $t$ mixture with Box-Cox transformation (flowClust), scale skewnormal (SSN) and skew-t (SST) mixtures, restricted skew-normal (rMSN) and skew- $t$ (rMST) mixtures, and unrestricted skew- $t$ mixture (uMST). All models are initialized by the partition obtained by the traditional $K$-means clustering. The algorithms stop when the stopping criterion meets the tolerance level of $1 e-5$. For more information about the behavior of different models, we refer the reader to the recent paper by Zhu and Melnykov (2016a), where a comprehensive simulation study is conducted to compare model performance.

Table 4 provides model-based clustering results. The number of parameters of the models are 20 (flowClust), 25 (SSN), 25 (SST), 25 (rMSN), 27 (rMST), and 27 (uMST). The computing times are 0.012, $1.553,4.737,0.024,0.136,3547.527$, respectively. The BIC values of the models are $3551.125,3576.886$, 3558.227, 3566.007, 3562.151, 3591.241. flowClust enjoys the lowest BIC value, which is still higher than that of Manly B. uMST yields the lowest number of misclassifications, which is as good as the full Manly mixture model and Manly forward model.

\section{Acknowledgement}

The research is partially funded by the University of Louisville EVPRI internal research grant from the Office of the Executive Vice President for Research and Innovation. 
Table 4: Classification tables for the AIS dataset. Rows and columns represent the true and estimated partitions, respectively. The bold font highlights correct classifications.

\begin{tabular}{|c|c|c|c|c|c|c|}
\hline \multirow[b]{2}{*}{ Group } & \multicolumn{2}{|c|}{ flowClust } & \multicolumn{2}{|c|}{$\underline{\text { SSN }}$} & \multicolumn{2}{|c|}{ SST } \\
\hline & 1 & 2 & 1 & 2 & 1 & 2 \\
\hline 1 & 99 & 1 & 99 & 1 & 99 & 1 \\
\hline 2 & 8 & 94 & 7 & 95 & 5 & 97 \\
\hline & & & & & & \\
\hline Group & 1 & 2 & 1 & 2 & 1 & 2 \\
\hline 1 & 100 & 0 & 99 & 1 & 98 & 2 \\
\hline 2 & 8 & 94 & 6 & 96 & 2 & 100 \\
\hline
\end{tabular}

\section{Bibliography}

E. Anderson. The Irises of the Gaspe peninsula. Bulletin of the American Iris Society, 59:2-5, 1935. [p181, 183]

C. Biernacki, G. Celeux, and G. Govaert. Choosing starting values for the EM algorithm for getting the highest likelihood in multivariate Gaussian mixture models. Computational Statistics and Data Analysis, 413:561-575, 2003. [p190]

G. Celeux and G. Govaert. A classification EM algorithm for clustering and two stochastic versions. Computational Statistics and Data Analysis, 14:315-332, 1992. doi: 10.1016/0167-9473(92)90042-E. [p180]

D. Cook and S. Weisberg. An Introduction to Regression Graphics. John Wiley \& Sons, New York., 1994. [p181, 187]

S. L. Crawford. An application of the laplace method to finite mixture distribution. Journal of the American Statistical Association, pages 259-267, 1994. doi: 10.1080/01621459.1994.10476467. [p192]

A. P. Dempster, N. M. Laird, and D. B. Rubin. Maximum likelihood for incomplete data via the EM algorithm (with discussion). Journal of the Royal Statistical Society B, 39:1-38, 1977. [p177]

R. A. Fisher. The use of multiple measurements in taxonomic poblems. The Annals of Eugenics, 7: 179-188, 1936. doi: 10.1111/j.1469-1809.1936.tb02137.x. [p181, 183]

N. A. Gillespie and M. C. Neale. A finite mixture model for genotype and environment interactions: Detecting latent population heterogeneity. Twin Res Hum Genet, 9(3):412-23, 2006. doi: 10.1375/ 183242706777591380. [p176]

S. X. Lee and G. J. McLachlan. Model-based clustering and classification with non-normal mixture distributions. Statistical Methods and Applications, 22(4):427-454, 2013. [p187, 189]

S. X. Lee and G. J. McLachlan. EMMIXuskew: Fitting unrestricted multivariate skew $t$ mixture models. Journal of Statistical Software, 55(12), 2014. doi: 10.1007/s11222-012-9362-4. [p176]

K. Lo, F. Hahne, R. R. Brinkman, and R. Gottardo. flowClust: a bioconductor package for automated gating of flow cytometry data. BMC Bioinformatics, 10(145), 2009. doi: 10.1186/1471-2105-10-145. [p176]

R. Maitra and V. Melnykov. Simulating data to study performance of finite mixture modeling and clustering algorithms. Journal of Computational and Graphical Statistics, 19(2):354-376, 2010. doi: 10.1198/jcgs.2009.08054. [p178]

B. F. J. Manly. Exponential data transformations. Biometrics Unit, 25:37-42, 1976. [p177]

G. J. McLachlan and K. E. Basford. Mixture Models: Inference and Applications to Clustering. Marcel Dekker, New York, 1988. doi: 10.2307/2348072. [p178]

G. J. McLachlan and T. Krishnan. The EM Algorithm and Extensions. Wiley, New York, 2nd edition, 2008. doi: 10.1002/9780470191613. [p177]

G. J. McLachlan and D. Peel. Finite Mixture Models. John Wiley \& Sons, New York, 2000. [p179, 184] 
V. Melnykov. On the distribution of posterior probabilities in finite mixture models with application in clustering. Journal of Multivariate Analysis, 122:175-189, 2013. doi: 10.1016/j.jmva.2013.07.014. [p179]

S. Michael and V. Melnykov. Finite mixture modeling of gaussian regression time series with application to dendrochronology. Journal of Classification, 33(3):412-441, 2016. doi: 10.1007/s00357-0169216-4. [p176]

B.-J. Park and D. Lord. Application of finite mixture models for vehicle crash data analysis. Accident Analysis E Prevention, 41(4):683-691, 2009. doi: 10.1016/j.aap.2009.03.007. [p176]

M. Prates, C. Cabral, and V. Lachos. mixsmsn: Fitting finite mixture of scale mixture of skew-normal distributions. Journal of Statistical Software, 54:1-20, 2013. doi: 10.18637/jss.v054.i12. [p176]

P. Schlattmann. Medical applications of finite mixture models. Springer, 2009. [p176]

G. Schwarz. Estimating the dimensions of a model. The Annals of Statistics, 6:461-464, 1978. [p179]

K. Wang, A. Ng, and G. McLachlan. EMMIXskew: The EM algorithm and skew mixture distribution, 2013. R package version 1.0.1. [p176]

J. H. Ward. Hierarchical grouping to optimize an objective function. Journal of the American Statistical Association, 58:236-244, 1963. [p186]

X. Zhu and V. Melnykov. Manly transformation in finite mixture modeling. Computational Statistics and Data Analysis, 2016a. doi: 10.1016/j.csda.2016.01.015. [p176, 195]

X. Zhu and V. Melnykov. ManlyMix: An R package for model-based clustering with manly mixture models, 2016b. R package version 0.1.9. [p176]

Xuwen Zhu

Department of Mathematics, The University of Louisville

Louisville, KY 40208

USA

xuwen. zhu@louisville. edu

Volodymyr Melnykov

Department of Information Systems, Statistics, and Management Science, The University of Alabama

Tuscaloosa, AL 35487

USA

vmelnykov@cba.ua.edu 